\title{
Tribonacci Quantum Cosmology: Optimal Non-Antipodal Spherical Codes \& Graphs
}

\author{
Angus McCoss \\ Kilbrandon House, PA34 4RA, UK \\ Email: ammcc0@outlook.com
}

How to cite this paper: McCoss, A. (2019) Tribonacci Quantum Cosmology: Optimal Non-Antipodal Spherical Codes \& Graphs. Journal of Quantum Information Science, 9, 41-97.

https://doi.org/10.4236/jqis.2019.91004

Received: February 11, 2019

Accepted: March 26, 2019

Published: March 29, 2019

Copyright () 2019 by author(s) and Scientific Research Publishing Inc. This work is licensed under the Creative Commons Attribution International License (CC BY 4.0).

http://creativecommons.org/licenses/by/4.0/

\begin{abstract}
Degrees of freedom in deep learning, quantum cosmological, information processing are shared and evolve through a self-organizing sequence of optimal $\star$, non-antipodal $\leftrightarrow$, spherical codes, ${ }_{\star}^{\star} C \subset S^{2}$. This Tribonacci
\end{abstract} Quantum Cosmology model invokes four ${ }_{\leftrightarrow}^{\star} C$ codes: 1-vertex, 3-vertex (great circle equilateral triangle), 4-vertex (spherical tetrahedron) and 24-vertex (spherical snub cube). The vertices are einselected centres of coherent quantum information that maximise their minimum separation and survive environmental decoherence on a noisy horizon. Twenty-four 1-vertex codes, $24 \times{ }_{\leftrightarrow}^{\star} C^{1}$, self-organize into eight 3 -vertex codes, $8 \times{ }_{\leftrightarrow}^{\star} C^{3}$, which self-organize into one 24 -vertex code, ${ }_{\leftrightarrow}^{\star} C^{24}$, isomorphic to dimensions of 24-spacetime and 12(2) generators of $S U(5)$. Snub cubical 24-vertex code chirality causes matter asymmetries and the corresponding graph-stress has normal and shear components relating to respective sides of Einstein's tensor equivalence $G_{\mu \nu}=\kappa \mathcal{T}_{\mu \nu}$. Cosmological scale factor and Hubble parameter evolution is formalized as an Ostwald-coarsening function of time, scaled by the tribonacci constant $(T \approx 1.839)$ property of the snub cube. The 24-vertex code coarsens to a broadband 4-vertex code, isomorphic to emergent 4-spacetime and antecedent structures in 24-spacetime metamorphose to familiar 4-spacetime forms. Each of the coarse code's 4-vertices has 6-fold parallelized degrees of freedom (conserved from the 24-vertex code), ${ }_{\leftrightarrow}^{\star} C^{4(6)}$, so 4 -spacetime is properly denoted 4(6)-spacetime. Cosmological parameters are formalized: $\mathrm{CMB} h=H_{0} / 100=T \log (3) / 3 \approx 0.674$, Distance Ladder $h=4 T \log (3) / 11 \approx 0.735, z_{d a}=3 \mathrm{e}^{1-3 / T}-1 \approx 0.596$, $\Omega_{b} h^{2}=\arccos (\sqrt{T / 2}) / 4 \pi \approx 0.023$ and $\gamma_{t}^{o} / \gamma_{t}^{c}=(T-1) / 2 \approx 0.420$. Due to 6 -fold parallelization, the total matter density parameter is 6-fold heavier than the baryon density parameter, 
$\Omega_{m}=6 \Omega_{b}=27 \arccos (\sqrt{T / 2}) / 2 \pi \log ^{2}(3) T^{2} \approx 0.302$. A torrent of information-equivalent energy downloads from 6 -fold faster 24-spacetime to 4(6)-spacetime. Consequent stress on 4(6)-spacetime causes it to resize its dynamic memory, expanding its cosmological scale. Ultimate coarsening of reality to ${ }_{\leftrightarrow}^{\star} C^{1}$, isomorphic to eternal time, is imminent for each observing agent in a Wheelerian participatory universe. DNA perhaps evolved from an $8 \times 3$-nucleotide primeval molecular code on the model's 24 shared dimensions.

\section{Keywords}

Deep Learning, Hubble, General Relativity, Spacetime, Particle Physics, Darwinism

\section{Introduction}

An original quantum cosmology model is introduced which extends the author's quantum intelligent cosmology research programme [1] [2] [3] [4]. The motivation is to further formalize the programme's original quantum foundational concepts whilst exploring encouraging new paths.

Let us commence by reflecting on a couple of inspiring quotes from John Archibald Wheeler, "Existence, what we call reality, is built on the discrete" and "... the world has at bottom an information-theoretic character" [5]. I propose that the discrete dimensions of our quantum information world are the most fundamental foundations of everything. A dimension is a structure that categorizes discrete information. Minimising computational time, a shared dimension makes economic computational use of that degree of freedom, as a Natural resource for the broadband parallel processing of distinguishable information.

This paper focuses on the Natural selection, evolution and function of shared dimensions in a deep learning quantum cosmology model. These shared dimensions are conformed dimensions in computer science, which ensure consistency between Nature's quantum information processing of its physics at macro, meso and microscopic scales; and ensure consistency spanning from deep evolutionary time, through observer lifetimes, to near-instantaneous causal physics.

The model presented proposes our deep learning quantum computational universe is founded on a common architecture of shared dimensions, defined by discrete and exceptional spherical codes, on a 2 -sphere, $S^{2}$. The shared dimensions are utilised by Nature to compute the entirety of physics, across the whole universe, at all scales. Nature's quantum computations at the cosmological scale can share dimensions with its computations at the Planck scale because the extreme difference in scale protects against detrimental crosstalk. Nature's two extreme scales come into perspective reality at the meso-scale of the sentient Wheelerian participatory observer. The information processed in these shared 
dimensions is parallelized, in coordinated superpositions, and decomposes into Nature's innumerable emergent physical phenomena, through observations, and described by effective theories. Fundamental information processing is impelled by Epistemic Drive, the Natural appetite for information selected for advantageous knowledge. This quantum cosmology (QC) model is underpinned by spherical codes, notably including the 24-vertex snub cubical spherical code, which implicitly involves the tribonacci constant, hence the moniker Tribonacci-QC.

The discrete dimensions of the spherical codes resemble the fine bristles in the puffball seed heads of the common dandelion flower (Taraxacum officinale) (Figure 1).

"No creature is fully itself till it is, like the dandelion, opened in the bloom of pure relationship to the sun, the entire living cosmos". Reflections on the death of a porcupine D. H. Lawrence (1925).

This paper is organized as follows:

Section 2 introduces the idea of a self-organizing sequence of optimal $\star$, non-antipodal $\leftrightarrow$, spherical codes, ${ }_{\leftrightarrow}^{\star} C \subset S^{2}$, in Tribonacci-QC. The codes configure degrees of freedom in quantum cosmological information processing, which are isomorphic to dimensions of 24-spacetime and to the 24 generators of the special unitary group of degree five, $S U(5)$. For background, the reader is referred to research on spherical codes [6], quantum self-organization [7] [8], geometrogenesis and quantum graphity [9] [10] [11], emergent spacetime [12], tropical geometry [13] [14], minimizing measures of the causal variational principle on the sphere [15] [16] [17] and the algebra of grand unified theories [18].

Section 3 reflects on quantum mechanics' profound relationship with the number 24. An inspiring lecture by mathematician John Baez [19] gives further context and deeper insight.

Section 4 considers the snub cubical characteristics of the 24-vertex optimal non-antipodal spherical code. This includes a discussion on the tribonacci constant, $T$, which derives from the third-order tribonacci recurrence sequence. The

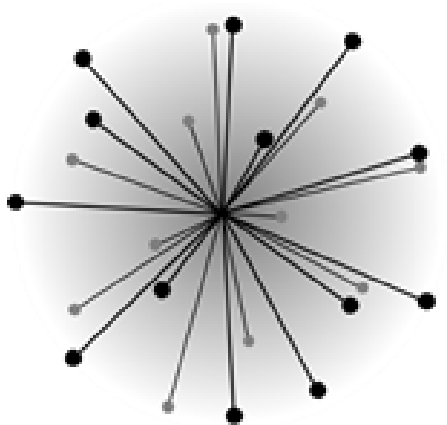

Figure 1. Tribonacci-QC: a quantum cosmology model dimensioned in optimal $\star$, non-antipodal $\leftrightarrow$, spherical codes, ${ }_{\leftrightarrow}^{\star} C \subset S^{2}$. In this illustration, radii connect the centre of the sphere with the 24 vertices (dots) of a spherical snub cube code, ${ }_{\leftarrow}^{\star} C^{24}$. The discrete dimensions of the model resemble the fine bristles in the puffball seed heads of the common dandelion flower (Taraxacum officinale). 
metric properties of the snub cube profoundly involve the tribonacci constant. The characteristic twist angle and chirality of the snub cube is also discussed. The properties of the snub cube are given in [20] [21]. Pertinent contemporary studies of the tribonacci sequence are given in [22] [23] and include those relating the tribonacci sequence to octonions and quaternions [24] [25], which in turn relate to fundamental interpretations of the standard model of particle physics [26] [27] [28] [29] [30].

Section 5 presents the model's cosmological scale factor evolution, $a(t)$, which is concisely formalized as an Ostwald-coarsening function of time, $t$, scaled by the tribonacci constant, $T$, where $a(t)=3^{(6 t)^{T / 3}-1}$ for $t<0$ and $a(t)=3^{t^{T / 3}-1}$ for $t>0$. The 6 -fold transition in the pace of time, at $t=0$, relates to geometrogenesis from 24-spacetime to 4(6)-spacetime (i.e. from a 24-vertex to a coarsened 4(6)-vertex optimal non-antipodal spherical code, as discussed in Section 2). The function takes as its origin, time $t=0$ and scale factor $a(t)=0$, relative to the spherical horizon datum. The present cosmological scale is defined $a(t) \rightarrow 1$, which is imminent, as $t \rightarrow 1$ for each observing agent in a Wheelerian participatory universe [5]. The Hubble parameter, deceleration-acceleration transition redshift and astronomical ladder-calibrated cosmological timescale are also formalized. Specifically, CMB $H_{0} / 100 \equiv h_{t \rightarrow 1} \equiv \dot{a} / a=T \log (3) / 3 \approx 0.67355$, Distance Ladder $h=4 T \log (3) / 11 \approx 0.73479$, redshift at the transition from a 24 -vertex to a $4(6)$-vertex code is defined $z_{24 \rightarrow 4(6)}=2$ (coeval with peak star formation rate density [31], quasar activity [32] [33], CMB lensing [34], ${ }^{6} \mathrm{Li}$ genesis by cosmic rays [35] [36] [37] [38] and exoplanetary abiogenesis [39] of Wheelerian participatory universe observers), and time of last zero cosmological scale is $t_{a=0}=13.8 \mathrm{Gyr}$, when calibrated by $t_{z=2}=10.4 \mathrm{Gyr}$ [40]. The redshift at the transition from deceleration to acceleration is $Z_{d a}=3 \mathrm{e}^{1-3 / T}-1 \approx 0.59607$ which compares well with supernovae observations [41] [42].

Section 6 presents this quantum cosmological model's formalization of baryon density as precisely one sixth of the total matter density, due to 6-fold parallelization at the transition from a 24-vertex to a coarsened 4(6)-vertex optimal non-antipodal spherical code. Specifically, $\Omega_{b} h^{2}=\arccos (\sqrt{T / 2}) / 4 \pi \approx 0.02287$ and $\Omega_{m}=6 \Omega_{b}=27 \arccos (\sqrt{T / 2}) / 2 \pi \log ^{2}(3) T^{2} \approx 0.30248$. This formalization derives from a proposed equivalence between the physical baryon density parameter, $\Omega_{b} h^{2}$, and the characteristic twist angle of the 24 -vertex snub cube, as a fraction of a rotational identity, $4 \pi$.

Section 7 considers the cosmographic distance ratio $\gamma_{t}^{o} / \gamma_{t}^{c}$ of the optical over cosmic microwave background tangential shear [43]. An equivalence in Tribonacci-QC is proposed, where $\gamma_{t}^{o} / \gamma_{t}^{c}=(T-1) / 2 \approx 0.41964$.

Section 8 considers astronomical observations [31]-[38] [44]-[56] which fit the Tribonacci-QC model and shows how the model circumvents cosmological singularity problems at the time of last zero cosmological scale $t_{a=0}$. This section also discusses cosmological evolution leading up to that event and considers 
this model in terms of John Archibald Wheeler's participatory universe [5]. Furthermore, DNA is proposed to have evolved from an $8 \times 3$-nucleotide (eight codon) primeval molecular code on the model's 24 shared dimensions.

Section 9 provides a summary of the paper and Section 10 gives conclusions.

\section{Optimal Non-Antipodal Spherical Codes}

In this model, a spherical code is a vertex set configured on the 2-sphere, $S^{2}$. A key objective of this current research is to identify a sequence of exceptional spherical codes which configure evolving degrees of freedom for deep learning, quantum cosmological, information processing by Nature. As such, they are isomorphic to evolving dimensions of spacetime and, in parallel, they are isomorphic to evolving shared dimensions of the Standard Model gauge group, $G_{S M}$, particularly as it relates to the special unitary group $S U(5)$.

In Tribonacci-QC degrees of freedom in quantum cosmological information processing:

- Create distinction (isolate crosstalk) [57] whilst computational dimensions are shared for parallel processing of information at cosmological and Planck scales;

- Order moments in time;

- Position locations in space;

- Frame spacetime events (located moments) [58];

- Constitute foundations for symmetrical quantum physics (Standard Model and dark sector) [59] [60];

- Accommodate and explain asymmetrical physics (arrow of time and e.g. matter-antimatter);

- Govern the quantum to classical transition [61];

- Enable cosmological expansion (Hubble phenomenon) [62];

- Relate to Einstein's general relativity tensor equivalence [63]; Additionally, the model embraces:

- Darwin's universal and deep learning algorithm (variational selective replication) [1] [64] [65] [66];

- Epistemic drive (Naturally selected information for advantageous knowledge) [1];

- Wheeler's participatory universe concept [5];

- Whitehead's perspective on occasions of experience [67];

- Astronomical observations.

To qualify as exceptional spherical codes that configure evolving degrees of freedom in this Tribonacci-QC model of quantum cosmological information processing with deep learning, the codes satisfy conditions:

1) Symmetrical vertex sets;

2) Few vertices ( $\leq 24)$;

3) Geometric compatibility to Naturally self-organize in an evolutionary sequence;

4) Non-antipodal (no diametrically opposite vertices); 
5) Maximise the minimum vertex separation (solving Tammes' problem) [68]-[73];

6) Chirality in the maximum vertex set of the sequence of exceptional spherical codes.

Succinctly, they are optimal non-antipodal spherical codes. Let us further consider each of these six above qualifying conditions:

1) Symmetrical vertex sets configure isomorphic degrees of freedom for symmetrical quantum physics, the symmetries in general relativity and for those in emergent Natural phenomena.

2) Optimal non-antipodal spherical codes have few vertices $(\leq 24)$ to create distinction between degrees of freedom and thus isolate crosstalk. Redundant or overcrowded vertices degrade information processing reliability and cause detrimental dimensional ambiguity; i.e. fewer channels are better for error free discretisation.

3) Geometric compatibility of optimal non-antipodal spherical codes to selforganize in sequence allows for Natural evolution towards spontaneous cosmological order. Consider analogous DNA which is geometrically comprised of nucleotide building blocks. Geometric compatibility facilitates self-organization. Kalinin et al. [74] seminally discuss self-organized criticality and pattern emergence through the lens of tropical geometry. I propose the geometry of shared dimensions in this Tribonacci-QC model will become more completely understood via application of the tools of tropical geometry [13] [14] which will lead to more profound formalizations of fundamental physics.

4) Non-antipodal vertex sets allow radial degrees of freedom in a spherical code, ${ }_{\infty}^{\star} C \subset S^{2}$, to have negative values without antipodal interference.

5) By maximising the minimum vertex separation optimal non-antipodal spherical codes effectively exhibit vertex-vertex "repulsion" which optimises their distinction and isolates degrees of freedom from detrimental crosstalk.

6) Chirality in the maximum vertex set of the sequence of exceptional spherical codes establishes a foundation for observed asymmetric physics (antimatter-matter) in our otherwise substantially symmetrical quantum physical cosmology. The maximum vertex set in Tribonacci- $Q C$ has 24 vertices in a spherical snub cubical configuration.

Applying the above conditions, rules and concepts, by elimination and Natural selection, the model invokes four optimal non-antipodal spherical codes: 1-vertex, 3-vertex (equilateral triangle on a great circle), 4-vertex (spherical tetrahedron) and 24-vertex (spherical chiral snub cube). The vertices are points of independent, coherent, einselected [61] [75] speciated information [1] [76], maximising separation and surviving environmental decoherence on a quantum noisy spherical horizon. Radii through the codes' points on the 2-sphere, $S^{2}$, configure isomorphic degrees of freedom in quantum cosmological information processing. These degrees of freedom are shared dimensions, that is they are utilised by the universe for its parallel quantum information processing and discre- 
tised at Planck and cosmological scales. Envisage points coincident with the code vertices on the 2-sphere, $S^{2}$, relate to Planckian computational discretisation, whilst vector heads of long radial vectors through the spherical code vertices relate to cosmological computational discretisation.

Spherical code evolution is a computationally intelligent, deep learning, self-organization process (Figure 2). Twenty-four 1-vertex codes self-organize into eight 3-vertex codes, which then self-organize into one 24-vertex code, isomorphic to the dimensions of 24-spacetime, in which antecedent quantum astrophysical structures emerge (albeit hard to completely visualise from and through subsequent 4(6)-spacetime). The 24 -vertex code is simultaneously isomorphic to the dimensions of quantum mechanics (Section 3).

Quantum self-organization on the spherical horizon locks at 24-vertex snub cubical packing, however information complexity inexorably continues to compound under Nature's epistemic drive [1]. The 24-vertex code coarsens into a broadband 4-vertex code, isomorphic to the dimensions of emergent 4(6)-spacetime. At this coarsening transition antecedent 24-spacetime quantum astrophysical structures metamorphose into their familiar 4(6)-spacetime quantum astrophysical structures. The transition from 24-spacetime to 4(6)-spacetime is a form of geometrogenesis, during which energy is conserved. Degrees of freedom are also conserved, though 6-fold parallelization occurs to accommodate the lower symmetry. This spontaneous phase transition may also be considered as a flash freezing via nucleation on the spherical horizon of the optimal non-antipodal codes, ${ }_{\leftrightarrow}^{\star} C \subset S^{2}$. Analogously, when water flash freezes to ice, its degrees of freedom instantly reduce and its scale factor steps up. When the rotationally symmetric liquid water state has degrees of freedom removed at a phase transition it crystallises into ice with a regular lattice structure and its volume expands. In Tribonacci-QC, the transition from 24-spacetime to 4(6)-spacetime causes space to expand.

Each of the vertices of the 4 -vertex code constitutes a quantum superposition of 6 parallelized corresponding isomorphic degrees of freedom (conserved from the prior 24-vertex code). Congruently, the total matter density parameter $\Omega_{m}$ is 6-fold heavier than the baryon density parameter $\Omega_{b}$ and an accompanying torrent of information-equivalent energy downloads from 6-fold faster 24-spacetime to emergent 4(6)-spacetime. Consequent stress on coarser 4(6)-spacetime causes it to resize its dynamic memory by eruptively expanding its cosmological scale (Section 5). For a related view on staged self-assembly of colloids see [73]. To deepen our understanding of the foregoing, we turn to graph theory and to Figures 3-5.

In Figure 3 we see illustrated a 24-vertex, 60-edge, snub cubical graph in its triangular perimeter, see also [77] (this graph is more commonly illustrated in its square perimeter [78] [79]). In its triangular perimeter, note the nested 1-gon (central vertex), in 5-gon (purple), in 8-gon (green), in 7-gon (red), in 3-gon (light blue) which has the following Diophantine relations, via their integer representations: 


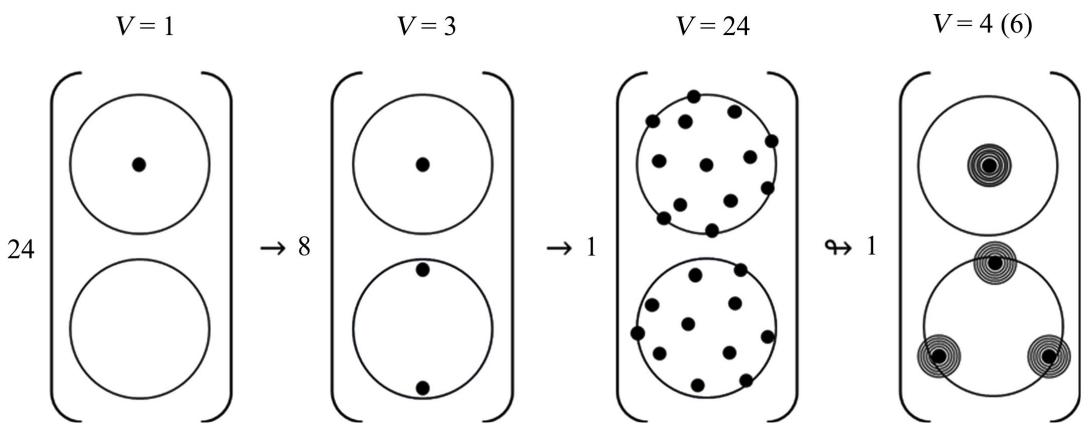

Figure 2. Optimal non-antipodal spherical codes. self-organization process, illustrated in stereographic projections. Far left: Twenty-four 1-vertex codes. Middle left: eight 3-vertex codes (equilateral triangle on a great circle). Middle right: one 24-vertex code (chiral, spherical snub cube). Far right: one 4 -vertex code (spherical tetrahedron) with 6 parallelized vertices superposed at each of the four tetrahedral vertices. The self-organization from twenty-four 1-vertex codes to eight 3-vertex codes and thence to one 24-vertex code achieves an optimal snub cubical packing. However, information complexity inexorably continues to compound, and the 24-vertex code coarsens into a broadband 4-vertex code.

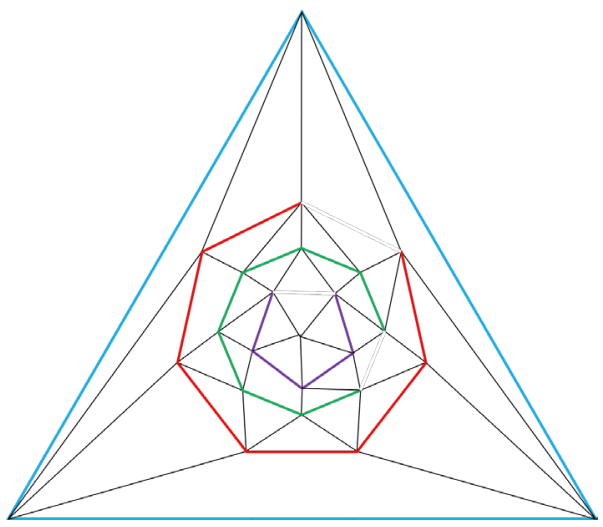

Figure 3. A 24-vertex, 60-edge, snub cubical graph in its triangular perimeter (this graph is more commonly illustrated in its square perimeter). In this triangular perimeter note the nested 1-gon (central vertex), in 5-gon (purple), in 8-gon (green), in 7-gon (red), in 3 -gon (light blue). The colours simply accentuate characteristic sub-graph shapes inherent within the snub cubical graph.
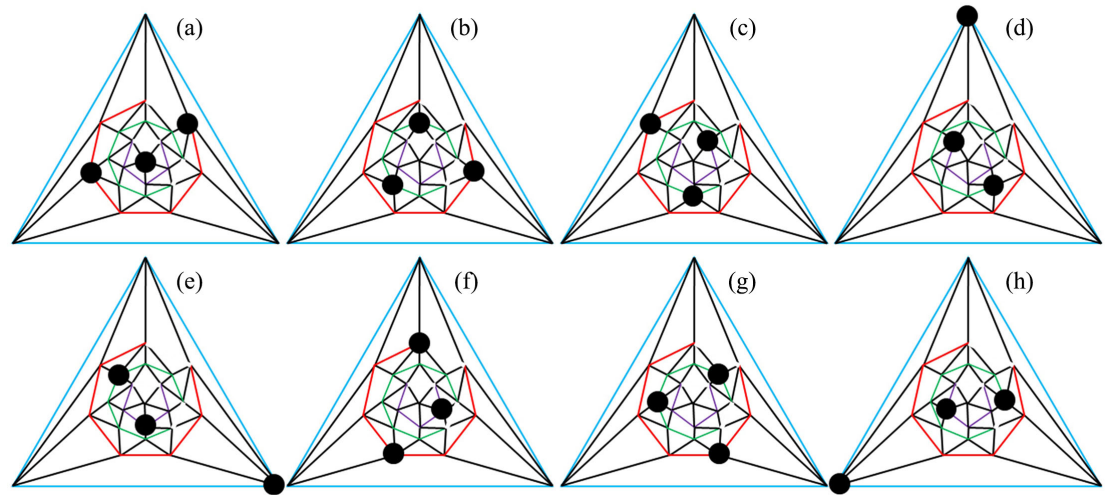

Figure 4. Eight snub cubical graphs, labelled (a-h), in (light blue) triangular perimeters (Figure 3) with eight 3-vertex sets (solid black dots) that self-organize into the 24-vertex set, isomorphic to the dimensions of 24-spacetime. 


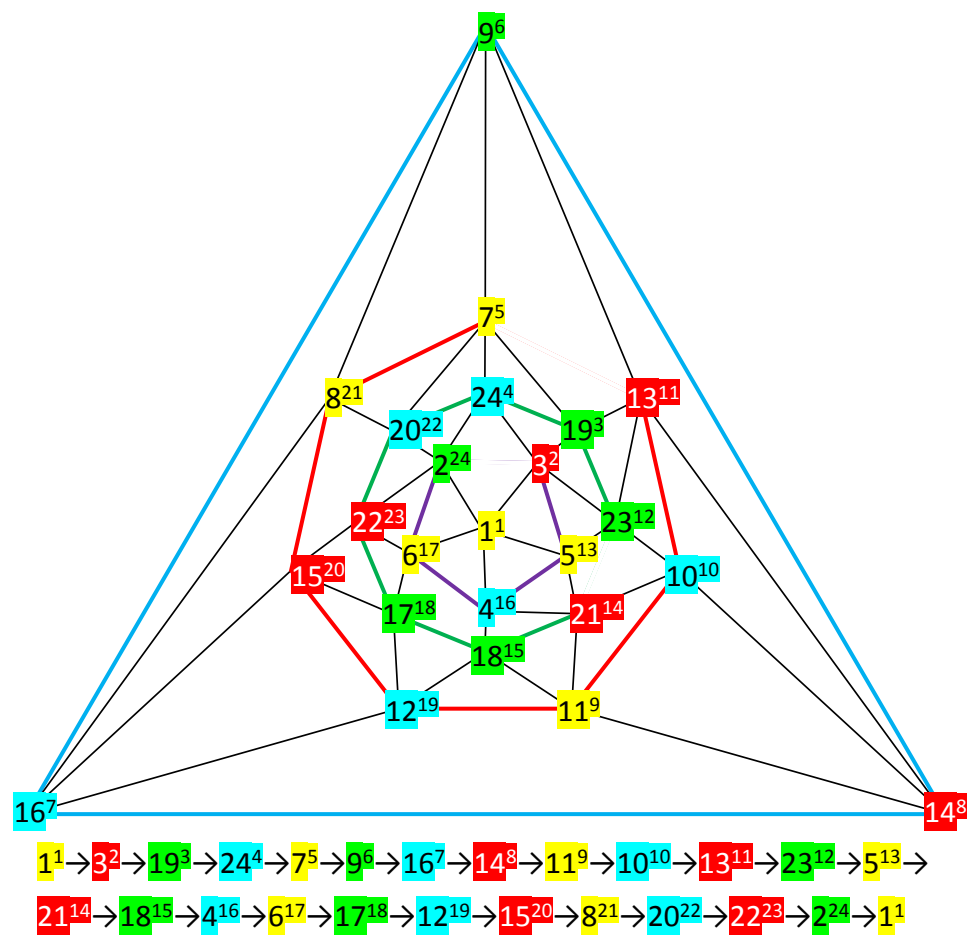

Figure 5. A 24-vertex, 60-edge, snub cubical graph in its triangular perimeter (Figure 3). The vertices are numbered according to the vertex adjacency list (Table 1) and the superscripts are index numbers in the example Hamiltonian super-cycle (2.5). Yellow labelled vertices are designated time-like $(t)$ and the red, green and blue labelled vertices are space-like, respectively $(x, y, z)$. The example Hamiltonian super-cycle (2.5) is included and is coloured consistently with the graph.

$$
\begin{gathered}
1+5+8+7+3=24 \\
1-5+8-7+3=0 \\
1^{2}-5^{2}+8^{2}-7^{2}+3^{2}=0
\end{gathered}
$$

The author presently offers no physical interpretation of this observation, though future research may link this pattern of integers to some fundamental properties in physics. I guess tropical geometric comparison of the snub cubical graph with the Standard Model gauge group, $G_{S M}$, particularly as it relates to the special unitary group $S U(5)$, may provide such enlightenment.

Illustrated in Figure 4, eight 3-vertex sets self-organize into the 24-vertex set, isomorphic to the dimensions of 24-spacetime. In the equivalent spherical codes, before ultimately settling into one, compact, snub cubical, 24-vertex code, the eight 3-vertex codes are equilateral triangles on great circles, i.e. they comprise eight 3-vertex optimal non-antipodal spherical codes that key together, interlocking to form the 24-vertex code. Considering the quantum self-organization of the isomorphic 24-vertex spherical code, it is noted that a small settling compaction is required to ultimately form a tightly fitting snub cubical optimal non-antipodal spherical code from eight such component equilateral triangles. The compacted and settled equilateral triangles, forming the self-organized snub 
cube, are finally circumscribed by (near-great) small circles with radius approximately 0.99688 of a corresponding unit radius spherical great circle. The settling compaction, $\Delta$, is given in (2.4) as a function of the tribonacci constant, $T$. Section 4 provides the geometric properties of the snub cube in terms of the tribonacci constant, from which this compaction factor is readily calculated.

$$
\Delta=\frac{2 \sqrt{T^{2}+\frac{1}{T^{2}}+T-\frac{1}{T}+2}}{\sqrt{3} \sqrt{\frac{1}{T^{2}}+1} \sqrt{\frac{T-3}{T-2}}} \approx 0.99688
$$

Shown in Figure 5, the 24-vertex, 60-edge, snub cubical graph is in its triangular perimeter (Figure 3 ) with vertices numbered and coloured. The numbers pertain to the following 5-valency, snub cubical graph vertex adjacency list (Table 1) [79]:

The snub cubical graph has Hamiltonian cycles. Let us consider the pertinent example Hamiltonian super-cycle, $\mathbf{H}_{\circlearrowright}$, depicted in Figure 5:

$$
\begin{aligned}
\mathbf{H}_{0}=\left\{1^{1}\right. & \rightarrow 3^{2} \rightarrow 19^{3} \rightarrow 24^{4} \rightarrow 7^{5} \rightarrow 9^{6} \rightarrow 16^{7} \rightarrow 14^{8} \rightarrow 11^{9} \rightarrow 10^{10} \\
& \rightarrow 13^{11} \rightarrow 23^{12} \rightarrow 5^{13} \rightarrow 21^{14} \rightarrow 18^{15} \rightarrow 4^{16} \rightarrow 6^{17} \rightarrow 17^{18} \\
& \left.\rightarrow 12^{19} \rightarrow 15^{20} \rightarrow 8^{21} \rightarrow 20^{22} \rightarrow 22^{23} \rightarrow 2^{24} \rightarrow 1^{1}\right\}
\end{aligned}
$$

which starts and recycles through the central vertex, numbered 1 (labelled yellow) and with superscript 1 referring to its index number in this example Hamiltonian super-cycle. The second vertex in the cycle is numbered 3 (labelled red) and with superscript 2 denoting its index as the second vertex in this example Hamiltonian super-cycle. The third vertex in the cycle is numbered 19 (labelled green) and with superscript 3 denoting its index as the third vertex in this example Hamiltonian super-cycle. The fourth vertex in the cycle is numbered 24 (labelled blue) and with superscript 4 denoting its index as the fourth vertex in this example Hamiltonian super-cycle, and so on through this cycle (2.5).

Table 1. Vertex adjacency list of 5-valency, snub cubical graph corresponding to Figure 5.

$\begin{array}{cc}1:(2,3,4,5,6) & 13:(9,10,14,19,23) \\ 2:(1,6,20,22,24) & 14:(9,10,11,13,16) \\ 3:(1,5,19,23,24) & 15:(8,12,16,17,22) \\ 4:(1,5,6,18,21) & 16:(8,9,12,14,15) \\ 5:(1,3,4,21,23) & 17:(6,12,15,18,22) \\ 6:(1,2,4,17,22) & 18:(4,11,12,17,21) \\ 7:(8,9,19,20,24) & 19:(3,7,13,23,24) \\ 8:(7,9,15,16,20) & 20:(2,7,8,22,24) \\ 9:(7,8,13,14,16) & 21:(4,5,10,11,18) \\ 10:(11,13,14,21,23) & 22:(2,6,15,17,20) \\ 11:(10,12,14,18,21) & 23:(3,5,10,13,19) \\ 12:(11,15,16,17,18) & 24:(2,3,7,19,20)\end{array}$


Note there are 6 sub-cycles comprising 4 vertices which start and end on a yellow labelled vertex, $V_{\text {yellow }}$ :

$$
V_{\text {yellow }}=\left\{1^{1}, 7^{5}, 11^{9}, 5^{13}, 6^{17}, 8^{21}\right\}
$$

A snub cube has 6 square faces and these 6 yellow labelled vertices each sit on a distinct square face (quadrilateral in the graph representation). The 6 corresponding vertex numbers from the above example Hamiltonian super-cycle (2.5) and (2.6) have the following Diophantine relations:

$$
\begin{gathered}
1+7+11+5+6+8=38=F_{\text {snub cube }} \\
1+7+11-5-6-8=0 \\
1+7+11=5+6+8
\end{gathered}
$$

where $F_{\text {snub cube }}$ is the number of faces of the snub cube. The author presently offers no physical interpretation of this observation, though future research may also link this pattern of integers to some fundamental properties in physics. Again, tropical geometry may provide the key.

The 6 yellow labelled vertices (2.6) are defined as isomorphic to time-like degrees of freedom, due to their model-assigned role in clocking the 6 successive ordered sub-cycles in the 24-vertex example Hamilton super-cycle (2.5).

Whereas in this example Hamiltonian super-cycle (2.5) the yellow labelled vertices, $1,7,11,5,6,8$, are designated time-like, the red, green and blue labelled vertices are space-like. The vertices labelled red are designated to the $x$ dimension of space and have vertex numbers $3,14,13,21,15,22$. The vertices labelled green are designated to the $y$ dimension of space and have vertex numbers 19,9 , $23,18,17,2$. The vertices labelled blue are designated to the $z$ dimension of space and have vertex numbers $24,16,10,4,12,20$.

Let us denote generic space-like vertices $(l)$, for length, whereas specific space-like vertices are $(x, y, z)$. Returning to Figure 4 , we now recognise that the eight 3-vertex sets, in this example Hamiltonian super-cycle (5), which self-organize into the snub cubical graph depicted in Figure 5, are categorised in the following summation:

$$
1\left(\begin{array}{c}
l \\
2 t
\end{array}\right)+4\left(\begin{array}{c}
2 l \\
t
\end{array}\right)+3\left(\begin{array}{c}
3 l \\
0
\end{array}\right) \Rightarrow 6\left(\begin{array}{c}
3 l \\
t
\end{array}\right)
$$

That is, for the example Hamiltonian super-cycle (2.5), one 3-vertex set with two time-like vertices, plus four 3 -vertex sets with one time-like vertex and three 3 -vertex sets which are entirely space-like, self-organize into a 24 -vertex set comprising six time-like vertices and eighteen $(6 \times 3)$ space-like vertices.

Figure 6 shows a 4(6)-vertex tetrahedral graph derived from a 6-fold chromatic parallelization of the vertices depicted in the 24 -vertex snub cubical graph in its triangular perimeter of Figure 5. The colours in the example Hamiltonian super-cycle (2.5) have self-organized into 4 monochromatic vertices (yellow is time-like $(t)$, whilst red $(x)$, green $(y)$ and blue $(z)$ are space-like). This transformation from the 24 -vertex graph to the $4(6)$-vertex tetrahedral multigraph represents a coarsening of the isomorphic dimensions of 24-spacetime to those of $4(6)$-spacetime. 


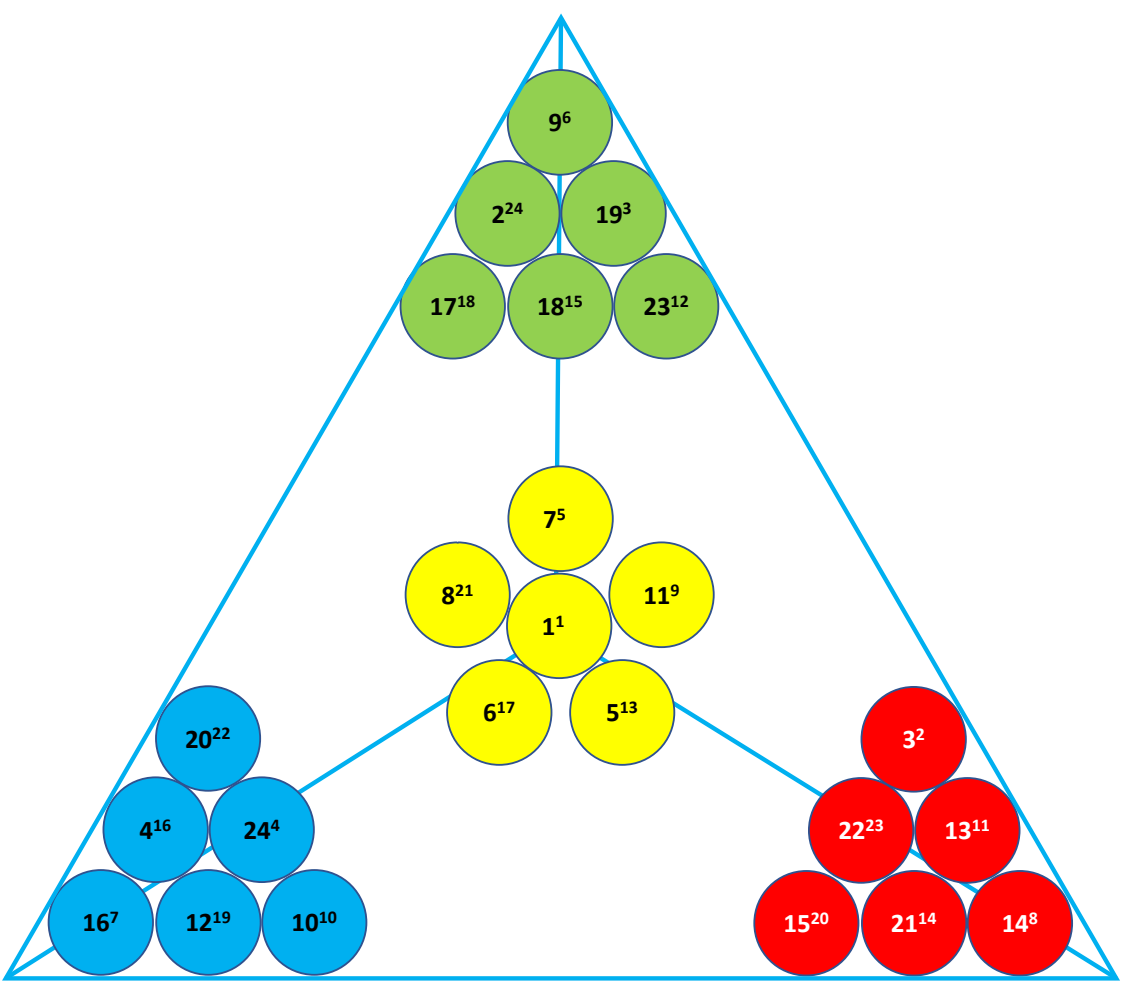

Figure 6. A 4(6)-vertex, 6(4)-edge, tetrahedral graph, derived from a 6-fold chromatic parallelization of the vertices depicted in the 24 -vertex snub cubical graph in its triangular perimeter (Figure 5).

The 6 time-like yellow labelled vertices (Figure 6) are isomorphic to 6 corresponding parallelized degrees of freedom and are collocated centrally, with vertex number 1 (tetrahedron apex). The red, green and blue labelled vertices are space-like and collocated at respective vertices of the graph's triangular perimeter (tetrahedron base). Referring to Figure 5 and Figure 6, the red labelled vertices are collocated with vertex number 14 , the green labelled vertices are collocated with vertex number 9 and the blue labelled vertices are collocated with vertex number 16 (Figure 7).

A simplified version of Figure 6 is shown in Figure 7 which depicts the chromatic parallelization of vertices of a tetrahedral graph, isomorphic to dimensions of 4(6)-spacetime. This is the result of coarsening the snub cubical graph shown in Figure 5. The arrows are directed from the example Hamiltonian super-cycle (2.5). The yellow dot $(t)$ represents 6 superposed time-like vertices. The red dot $(x)$ represents 6 superposed space-like vertices. The green dot $(y)$ represents 6 superposed space-like vertices. The blue dot $(z)$ represents 6 superposed space-like vertices. I now deconstruct Figure 6 and Figure 7 , to further illustrate the workings of the example Hamiltonian super-cycle (2.5).

Adopting the layout of Figure 7, I now exhibit, in Figure 8, a deconstruction of the example Hamiltonian super-cycle (2.5) into its 6 sub-cycles. A rotating pattern is evident. 


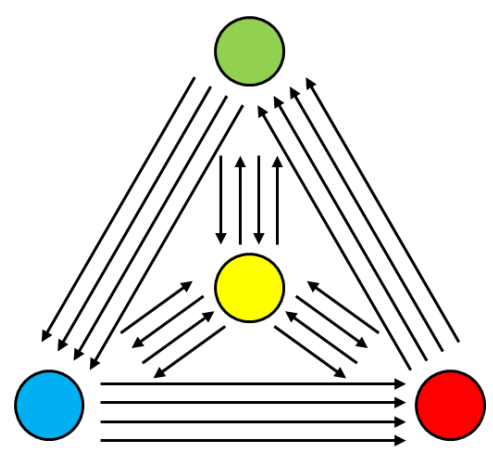

Figure 7. Simplified version of Figure 6 depicting the chromatic parallelization of vertices of a tetrahedral graph, isomorphic to dimensions of 4(6)-spacetime. Yellow dot $(t)$ represents 6 superposed time-like vertices. Red dot $(x)$ represents 6 superposed space-like vertices. Green dot $(y)$ represents 6 superposed space-like vertices. Blue dot (z) represents 6 superposed space-like vertices. The arrows are directed from the example Hamiltonian super-cycle (2.5).
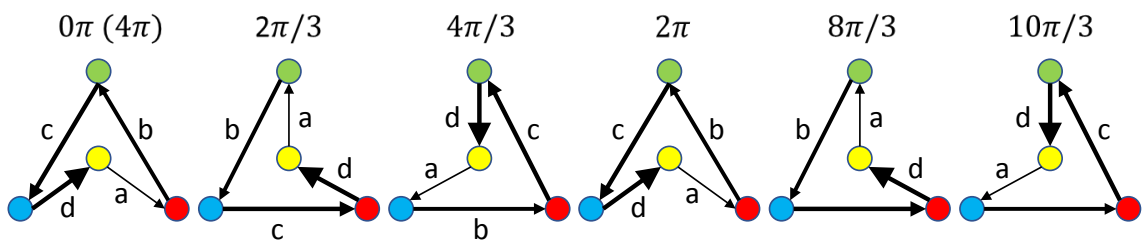

\section{$1,3,19,24,7 \quad 7,9,16,14,11 \quad 11,10,13,23,5 \quad 5,21,18,4,6 \quad 6,17,12,15,8 \quad 8,20,22,2,1$}

Figure 8. 24-vertex, snub cubical, example Hamiltonian super-cycle (2.5) (Figure 6 and Figure 7), is displayed here, coarsened and parallelized. It is deconstructed to show its 6 tetrahedral subgraph sub-cycles, which clock a $4 \pi\left(720^{\circ}\right)$ identity circuit, in 6 ordered turns of $2 \pi / 3\left(120^{\circ}\right)$, around the yellow-labelled time-like vertex. Each of the 4 tetrahedral vertices (isomorphic to 1-time dimension and 3-space dimensions) comprises 6 parallelized corresponding degrees of freedom.

In Figure 8 we can see the example Hamiltonian super-cycle (2.5), (Figure 5), coarsened and 6-fold parallelized, as in Figure 7. It is deconstructed to show its 6 tetrahedral subgraph sub-cycles, which register a $4 \pi\left(720^{\circ}\right)$ identity circuit, in 6 ordered turns of $2 \pi / 3\left(120^{\circ}\right)$, around the yellow-labelled time-like vertex, in a rotating pattern. Each of the 4 tetrahedral vertices (isomorphic to 1-time dimension and 3-space dimensions) comprises 6 parallelized corresponding degrees of freedom.

We may think of the above discussion and Hamiltonian super-cycle (2.5), which concludes with Figure 8, as formalizing a kind of cellular automaton, or vector space analysis, that frames spacetime events (located moments) [58] [80].

A complementary path to map the 24 -vertex set snub cube to the 4 -vertex set tetrahedron is via the 24-cell and the binary tetrahedral group [81] of order 24 (Section 3). The binary tetrahedral group is the group of units in the ring of Hurwitz integers, with its 24 units given by all sign combinations of

$$
\left\{ \pm 1, \pm i, \pm j, \pm k, \frac{1}{2}( \pm 1 \pm i \pm j \pm k)\right\}
$$


All 24 units have absolute value 1 and lie in the unit quaternion group $S p(1)$. The convex hull of the 24 elements in 4-dimensional space forms the 24-cell, which is a convex regular 4-polytope.

Figure 9 illustrates the same snub cubical graph as shown in Figure 5, with the same vertex number labels and same sequential indices, as per the example Hamiltonian super-cycle (2.5). Here, however, this configuration of the snub cubical graph accentuates a decomposition of the 24-vertex set into four 6-gons (solid black edges). Whilst the four 6-gons in Figure 9 might establish a definition of four vertices of an isomorphic tetrahedral graph, there is no obvious computational parallelization benefit, nor direction, within any of the 6-gons, hence the parallelized configuration depicted in Figures 6-8 is Naturally selected in the Tribonacci-QC model.

Summarising this Tribonacci-QC, optimal non-antipodal spherical code, model thus far, Figure 2 illustrates that twenty-four 1-vertex codes self-organize into eight 3-vertex codes (Figure 4, in graph representation), which then selforganize into one 24-vertex code (Figure 5, in graph representation), isomorphic to dimensions of 24-spacetime. This 24-vertex code then coarsens into a broadband 4(6)-vertex code that has a spherical tetrahedral arrangement with 6 -fold parallelization of vertices. The 4(6)-vertex code is isomorphic to the dimensions of 4(6)-spacetime. The vertex sets in Figure 2 are optimal non-antipodal spherical codes which are einselected [61] centres of coherent quantum information that maximise their minimum separation and survive environmental decoherence on a noisy spherical horizon. As such they configure the isomorphic degrees of freedom of emergent spacetimes and the dimensions, or generators, of the $S U$ (5) group (Section 3).

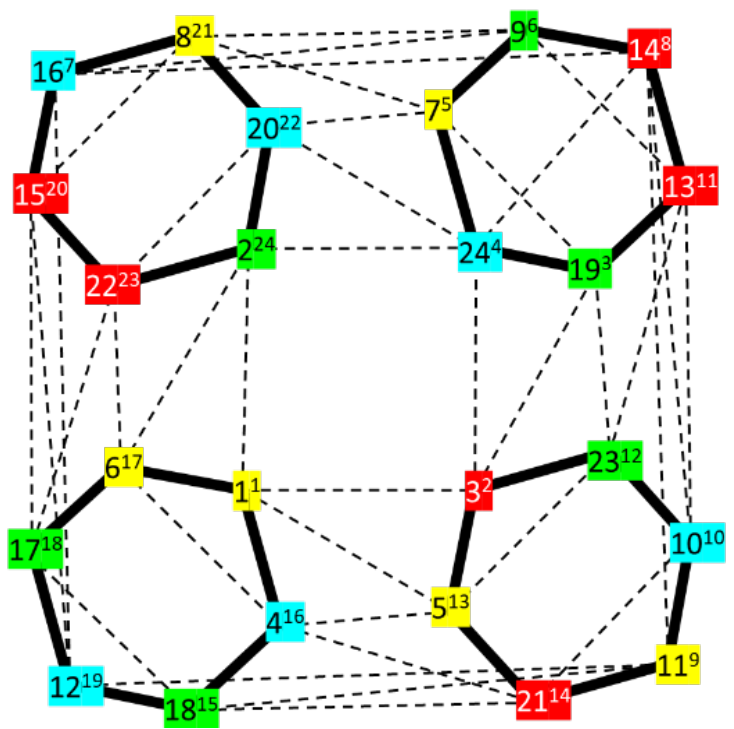

Figure 9. The same snub cubical graph as shown in Figure 5, with the same vertex number labels and same sequential indices, as per the example Hamiltonian super-cycle (2.5). Here, however, this configuration of the snub cubical graph accentuates a decomposition of the 24-vertex set into four 6-gons (solid black edges). 
The 24-vertex code has 6 time-like vertices, one per each of the four square faces of the snub cubical configuration. Quantum cosmological information processing, with deep learning, executes 6-fold faster through its resource of 6 distinct time-like degrees of freedom which order moments in time. Clocks in 24-spacetime advance 6-fold faster than in the subsequent coarser 4(6)-spacetime.

6-fold parallelization of 1 time-like and 3 space-like degrees of freedom in coarser 4(6)-spacetime provides Nature with quantum cosmological information processing which is 6-fold deeper and emerges 6-fold heavier than classically apparent. It is proposed in the model, that this 6-fold parallelization is manifest in the classical realm via 4(6)-dimensional general relativity and in the parallel processed quantum microscopic realm via 24 , or more strictly, 12(2)-dimensional quantum physics, with both sets of dimensions being referenced to the spherical horizon.

Although a hard problem, we can begin to imagine extending 4(6)-dimensional (3-space, 1-time) general relativity back into prior 24-spacetime, with its finer-grained 24-dimensional (18-space, 6-time) quantum general relativity.

\section{Quantum Mechanics with 12(2) = 24 Degrees of Freedom}

4-dimensional spacetime and corresponding general relativity is very well established in modern physics. It has also been related to a 2-dimensional horizon, via the holographic principle [82] [83] [84] and AdS/CFT correspondence [85].

6 -fold higher degrees of freedom in 12(2) = 24-dimensional quantum physics also feature in established research and models of particle physics. According to quantum mechanics, a harmonic oscillator that vibrates with frequency $\omega$ can have quantized energy

$$
\frac{1}{2} \omega,\left(1+\frac{1}{2}\right) \omega,\left(2+\frac{1}{2}\right) \omega,\left(3+\frac{1}{2}\right) \omega,\left(4+\frac{1}{2}\right) \omega, \cdots
$$

in units where Planck's constant equals 1 . The lowest energy is $\frac{1}{2} \omega$, (not zero due to the uncertainty principle), which is the ground state of the oscillator.

Euler observed that the surprizing sum of the Natural numbers to infinity is

$$
1+2+3+4+\cdots=-\frac{1}{12}
$$

It is now well known that the zeta function of -1 has the same value

$$
\zeta(-1)=-\frac{1}{12}
$$

A string oscillating in one direction is the same as an infinite collection of oscillators with frequencies

$$
\omega=1,2,3,4, \cdots
$$

and their ground states add, so the ground state energy of the string is given by the zeta function regularization 


$$
\frac{1}{2}(1+2+3+4+\cdots) \omega=\frac{1}{2} \zeta(-1)=-\frac{1}{24} \omega
$$

This is one of Nature's many fundamental mathematical incidences of the number 24 and some other occurrences are summarised as follows.

The Leech lattice $\Lambda_{24}$ is one of the most profound. It can be defined using octonion triplets and the Lie group $E_{8}$ [86] [87] which directly relates it to the mathematical foundations of particle physics [26] [27] [28] [29] [30]. The Leech lattice $\Lambda_{24}$ is the densest hypersphere packing in 24 dimensional Euclidean space and is mathematically related to the binary Golay code, which is a renowned error-correcting code capable of efficiently detecting 4 errors and correcting up to 3 errors in each 24 -bit word.

The Dedekind eta function, $\eta(\tau)$, is also mathematically related to the Leech Lattice, where

$$
\eta(\tau)=q^{\frac{1}{24}} \prod_{n=1}^{\infty}\left(1-q^{n}\right)
$$

and $q \equiv \mathrm{e}^{2 \pi i \tau}$, from number theory, and $\tau$ is any complex number where $\operatorname{Im}(\tau)>0$. The exponent $1 / 24$ is a consequence of the relationship with $\Lambda_{24}$ and furthermore the Dedekind eta function, $\eta(\tau)$, is intrinsically related to Virasoro algebra, conformal field theory and string theory.

Chapline's work on a matrix non-linear Schrödinger equation living on a Lorentzian extension of the 24-dimensional Leech lattice, as a potential framework for a quantum theory of gravity and elementary particles in 4-dimensions, is also insightful [88].

Also related to the Leech lattice, so called monstrous moonshine (moonshine theory) is the surprising relationship between modular functions (particularly the $j$-function) and the monster group [89], again connecting the number 24 to string theory.

Parenthetically, the Higgs field in $S U(5)$ is 24-dimensional. I return to SU (5) theory below.

Indeed, Nature's above proclivity towards the number 24 was wonderfully explored by Baez in a Rankine Lecture in 2008, and a recording can now be viewed online [19]. The numerical connections between the 1 , to 3 , to 8 -vertex set quantum self-organization in this quantum cosmology model, Tribonacci-QC, and the profound occurrences of unitarity, triality and the octonions in mathematical physics and quantum physics, is noteworthy.

Whilst the Tribonacci-QC model is not presented in the language of bosonic string theory, an association is conjectured through the fundamental relationship of both to 24 degrees of freedom. Physical degrees of freedom of the bosonic string are given as 24 transverse coordinates relative to a worldsheet, whereas the 24-vertex set, and its coarser form the 4(6)-vertex set, optimal non-antipodal spherical codes of the Tribonacci-QC have 24 degrees of freedom relative to their spherical horizon.

In the rest of this section we examine the shared dimensions of the Tribonac- 
ci-QC model by considering possible correspondences between shared dimensions of spacetime and the dimensions of particle physics. In doing so, we explore paths framed by the Tribonacci-QC model which potentially lead to the discovery of long-sought fundamental correspondences between general relativity and quantum mechanics. Again, tropical geometry may provide useful tools to further formalize the correspondences.

Referring to Figure 10 and recent work by Furey [90] and others, there are 24 generators of $S U(5)$ [18] [91] ladder symmetries which split into two types: 12 mixing and 12 non-mixing. Also recall (3.2) and (3.3) above, where the significance of this integer, twelve, is emphasised.

Consider non-mixing generators analogous to the Naturally selected optimal non-antipodal spherical codes in this Tribonacci-QC model. Non-mixing and non-antipodal characteristics both bestow discrete identity. Tribonacci- $Q C$ considers 12(2)-dimensional quantum physics occurring in deep 24-spacetime, thence and presently parallelized in coarser 4(6)-spacetime.

The deselected 12 mixing generators (dark boxes placed in Figure 10), responsible for proton decay, are excluded by Furey because in her division algebraic construction the two types of ladder operators are clearly algebraically distinct. The Naturally selected non-mixing generators (white boxes placed in Figure 10) do not mix the two types of ladder operators. Transitions leading to proton decay are expected by Furey to be blocked, given they coincide with forbidden transformations which would incorrectly mix distinct algebraic actions.

The 12 Naturally selected non-mixing generators comprise 8 which generate $S U(3)_{C}, 3$ which generate $S U(2)_{L}$ and 1 which coincides with hypercharge Y. In the Tribonacci-QC model, the tetrahedral 4(6)-spacetime state emerges from the coarsening of the snub cubical 24-spacetime state. In a quantum cosmological computational process, involving parallel processing in shared dimensions, the tetrahedral spherical code configuring isomorphic degrees of freedom also accommodates a parallelization of the 24 generators of $S U(5)$ and Naturally selects the 12 generators of the Standard Model gauge group, $G_{S M}$.

The red-green-blue base of the tetrahedral graph (Figure 6 and Figure 7) accommodates the spatial dimensions of spacetime and, via shared dimensions, in $G_{S M}$ each of these 3 vertices also accommodates 2 non-mixing generators of $S U(3)_{C}$ and a single indivisible non-mixing generator of $S U(2)_{L}$ (Figure 10). The yellow apex of the tetrahedral graph (Figure 6 and Figure 7) accommodates the time dimensions of spacetime and in $G_{S M}$ this apical vertex also accommodates 2 non-mixing generators of $S U(3)_{C}$ and a single indivisible non-mixing generator of $U(1)_{Y}$ (Figure 10).

The degree of parallelization through dimensional reduction of the 24 generators of $S U(5)$ at each vertex has a limiting lowest common denominator of 2 , set by the single indivisible generators at each vertex (an $S U(2)_{L}$ generator at each vertex on the triangular base and a $U(1)_{Y}$ generator at the apical vertex). The deselected 12 mixing generators are located virtually at their corresponding vertices, however they are computationally overwritten in the Tribonacci-QC 


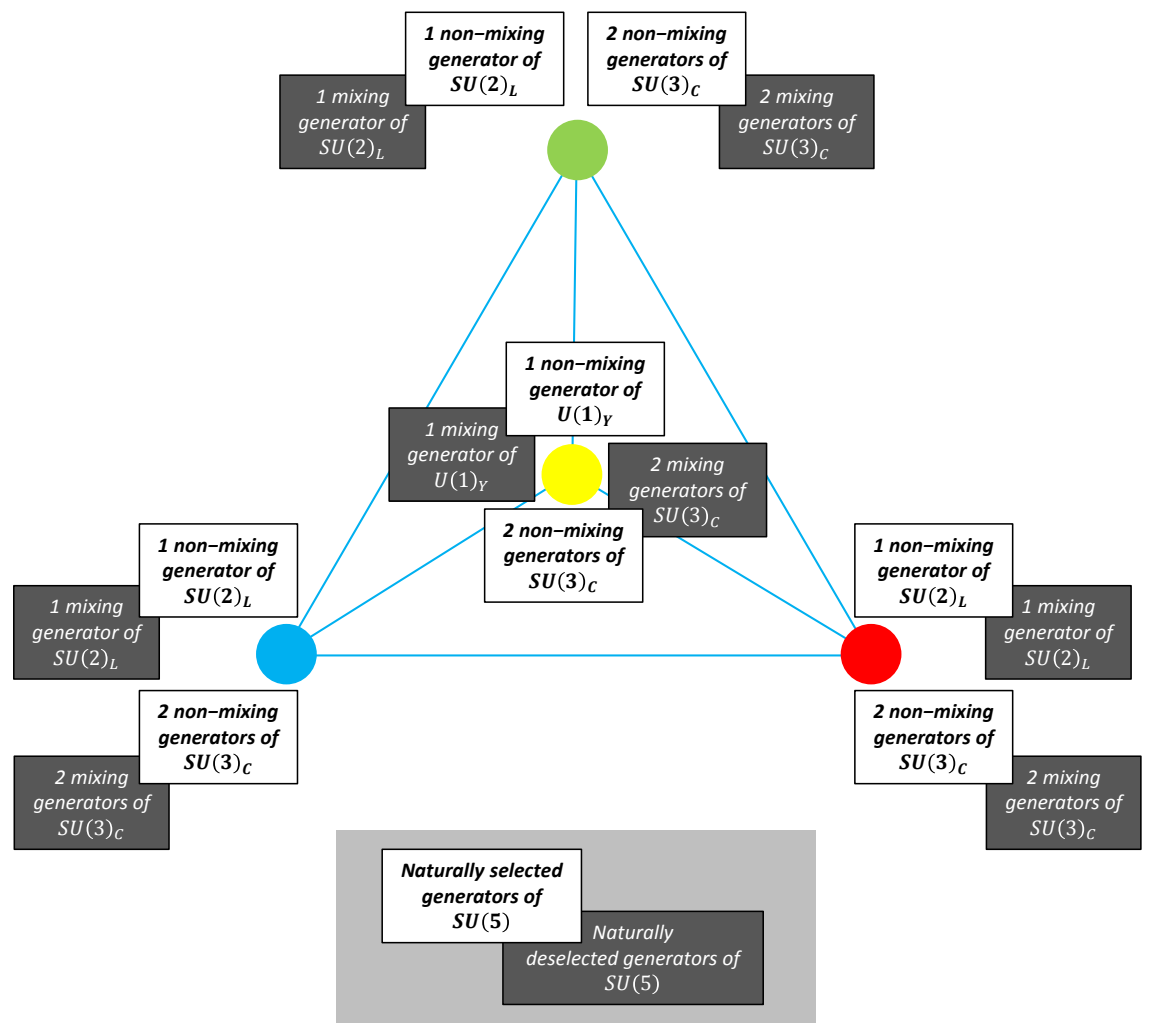

Figure 10. Based on the tetrahedral graph representation (Figure 6 and Figure 7) of the corresponding tetrahedral spherical code, which is isomorphic to the dimensions of $4(6)$-spacetime, this diagram illustrates a possible parallel configuration of $12(2)=24$ generators of $S U(5)$ ladder symmetries which are split into two types: 12 Naturally deselected mixing generators (dark boxes) and 12 Naturally selected non-mixing generators (white boxes). Tribonacci-QC considers 12(2)-dimensional quantum physics occurring in deep 24-spacetime, thence and presently in coarser 4(6)-spacetime.

model by the Naturally selected, principal, 12 non-mixing generators. Expanded mathematical definitions of the mixing and non-mixing generators are given by Furey [90].

Broadly, the Standard Model consensus theory of particle physics, is an $S U(3) \times S U(2) \times U(1)$ gauge theory which is experimentally well established at presently probed scales. The Standard Model gauge groups

$S U(3) \times S U(2) \times U(1)$ fit in the $S U(5)$ group of special unitary transformations acting on 5 complex variables.

Alleviating its proton decay problem, Furey [90] retains highly attractive features of $S U(5)$ theory. She considers the Standard Model represented by $G_{s m}=S U(3)_{C} \times S U(2)_{L} \times U(1)_{Y} / \mathbb{Z}_{6}$ (note the $\mathbb{Z}_{6}$ is commonly modded out) and finds that the $S U(5)$ ladder symmetries then reduce to it, where ladder operators arise from the division algebras $\mathbb{R}$ (real numbers), $\mathbb{C}$ (complex numbers), $\mathbb{H}$ (the quaternions), and $\mathbb{O}$ (the octonions).

Koca et al. [92] formally describe chiral polyhedral, including the snub cube, as derived from coxeter diagrams and quaternions. Future studies may connect their work to that of Furey [90] and to others, including Cerda-Morales who 
formalizes (snub cubical) tribonacci-type octonions sequences and describes their properties [24] see Section 4.1 below.

In graph theory, the snub cubical graph is a quintic graph where all vertices have degree 5. See the vertex figure in Figure 11. I conjecture that a fundamental connection exists between the 5 complex variables in $S U(5)$ and the 5 edges incident to each of the 24 vertices of the snub cubical graph of this Tribonacci-QC model (another lead for tropical geometricians). SU(5) has 24 corresponding generators, where $5 \times 5-1=24$; twelve of which are Naturally selected and twelve of which are virtual (Naturally deselected). Recall, Tribonacci-QC considers 12(2)-dimensional quantum physics occurring in deep 24-spacetime, thence and presently parallelized in coarser 4(6)-spacetime.

Alternatively, let us consider the representation of the snub cube, central to Tribonacci- $Q C$, as two 12 -vertex sets, one corresponding to the 12 Naturally selected generators and the other corresponding to the 12 virtual (Naturally deselected) generators. In Figure 12, we see the 24-vertex snub cubical graph configured in two sub-graphs, each comprising 12 vertices (one with vertices coloured blue and numbered 1, 2, 3, 4, 5, 6, 7, 19, 20, 22, 23, 24; and the other with vertices coloured green and numbered $8,9,10,11,12,13,14,15,16,17,18,21$ ). The number of coupling-edges, connecting the two sub-graphs, is minimized to twelve (in Figure 12 these 12 coupling-edges are coloured cheddar-orange, like stringy cheese between two halves of a grilled cheese sandwich!).

Each of the 12-vertex sub-graphs (blue subgraph and green subgraph) has 24 edges. The vertex numbering is consistent with the adjacency list in Table 1. Depicting an alternative configuration to Figure 10, these two 12-vertex sub-graphs in Figure 12 represent two sets of 12 generators, selected (non-mixing) and deselected (mixing), in $S U(5)$. Let us consider the green vertices selected, and the blue vertices deselected.

Consider Figure 13, which shows the snub cubical graph depicted in Figure 12, however here it is constructed within a triangular perimeter, like that in Figure 5. We see the blue vertices representing Naturally deselected mixing generators of $S U(5)$ are located centrally, clustered around the apex of the corresponding (coarsened) tetrahedral graph configuration (Figure 7). Whereas the green vertices, representing Naturally selected non-mixing generators of $S U$ (5) circumscribe the blue apex in a corresponding base-of-tetrahedral graph configuration. In Figure 7 the apex is defined to be time-like, whereas the basal triangle is space-like, with each vertex of the basal triangle corresponding to a dimension of 3-space. From this definition of blue and green vertices, we see that the green, Naturally selected 12 generators of $S U(5)$, are disposed to be more space-like in terms of vertex adjacency (Figure 13), although they are both spacelike and time-like in terms of the Hamiltonian super-cycle (2.5) depicted in Figure 5 and Figure 6.

See also Section 8.2, where Figure 13 is interpreted as a quantum mechanical configuration of a Planck star at the core of a black hole, where the enclosed, 
apical, time-like vertices $1,2,3,4,5,6,7,19,20,22,23,24$, correspond to quantum mechanics concealed within the black hole.

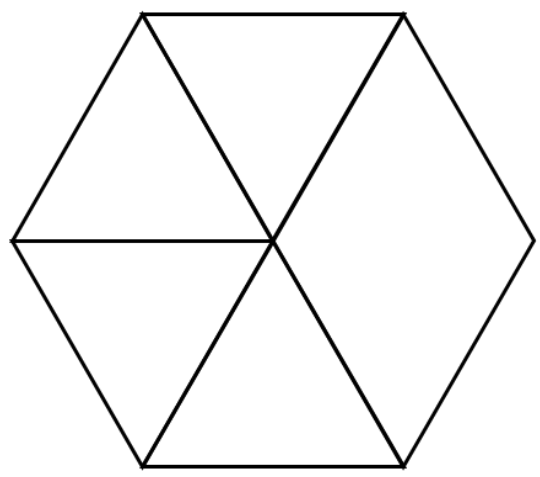

Figure 11. Vertex figure of the snub cube (5-valent Archimedean polyhedron).

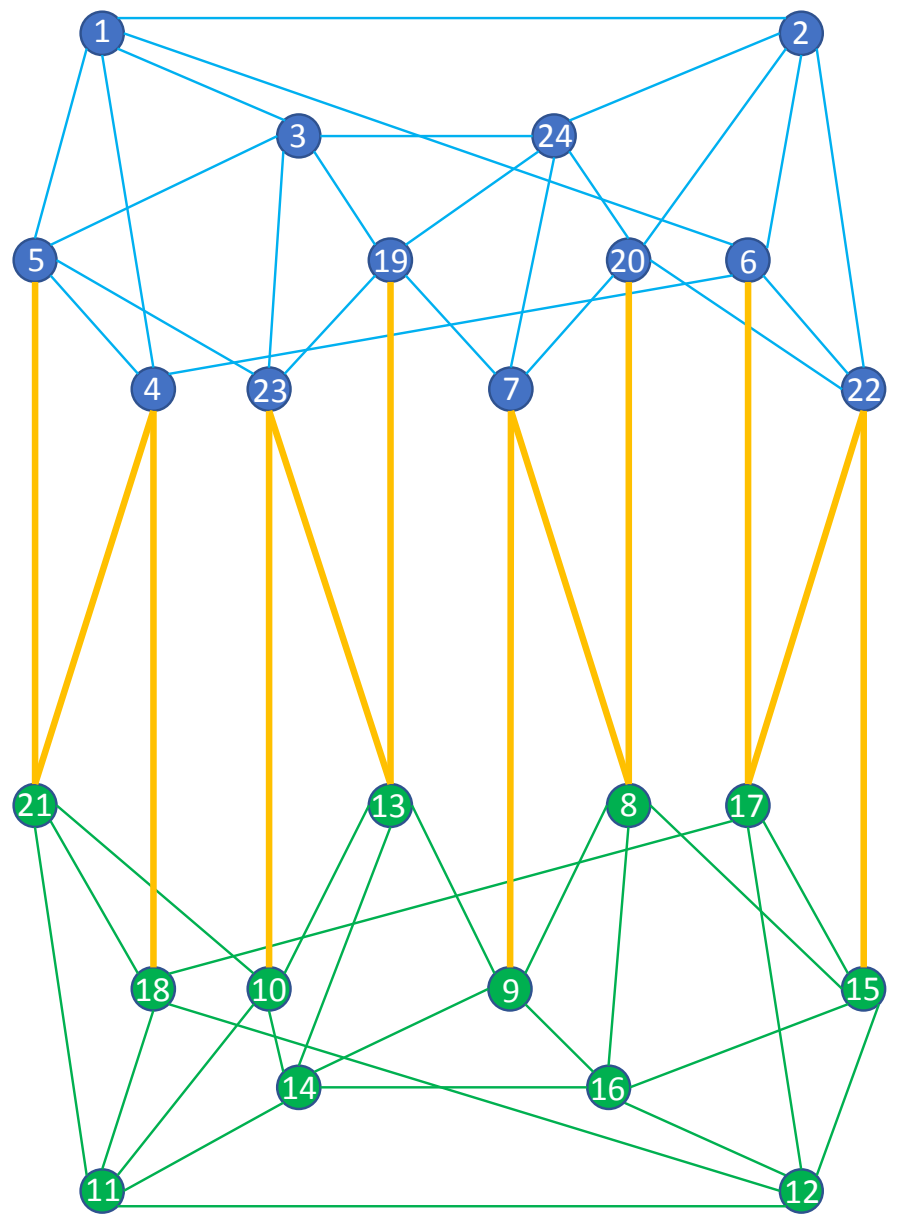

Figure 12. 24-vertex snub cubical graph configured in two sub-graphs, each comprising 12 vertices (one with vertices coloured blue and numbered 1, 2, 3, 4, 5, 6, 7, 19, 20, 22, 23, 24; and the other with vertices coloured green and numbered $8,9,10,11,12,13,14,15$, $16,17,18,21)$. The number of coupling-edges connecting the two sub-graphs is minimized to twelve (coloured cheddar-orange, like stringy cheese between two halves of a grilled sandwich!). Each of the 12-vertex sub-graphs (blue and green) has 24 edges. Vertex numbering is consistent with the adjacency list in Table 1. 


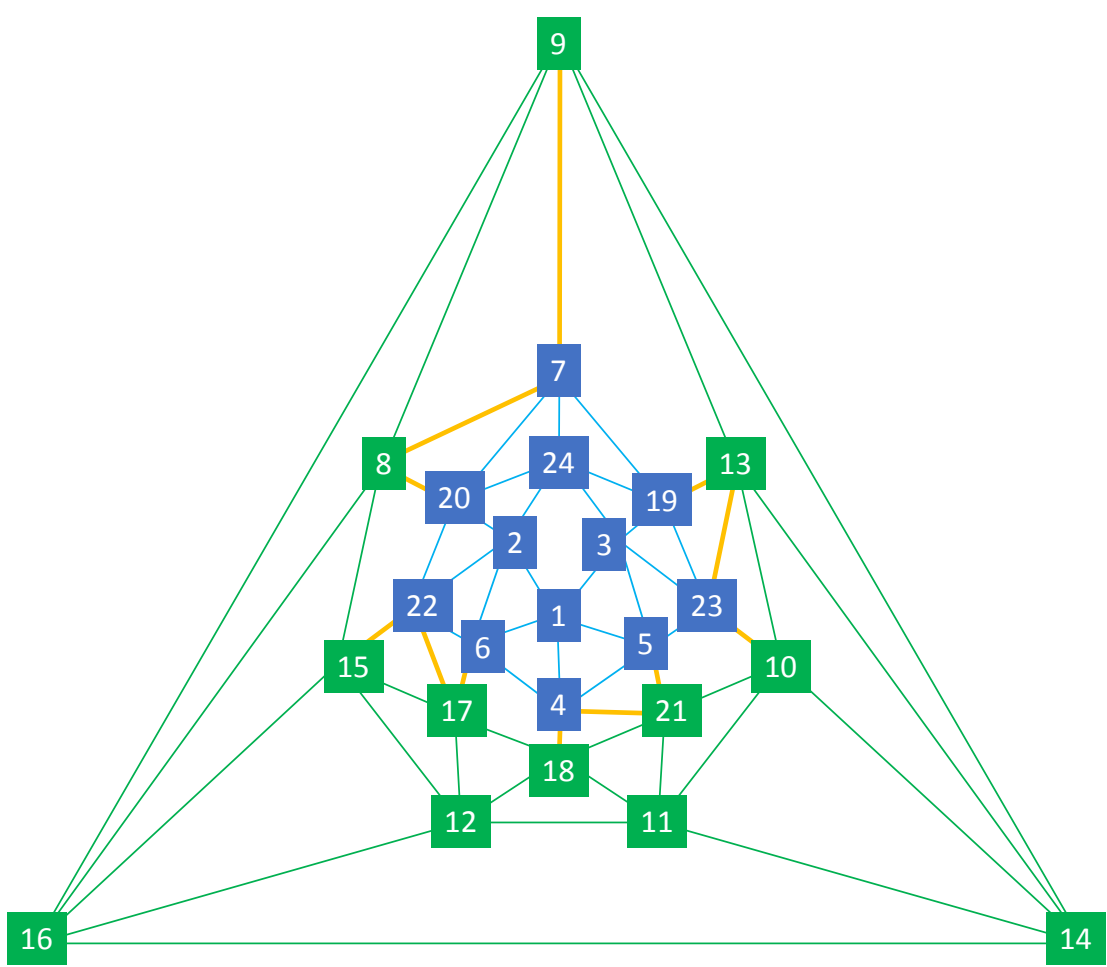

Figure 13. The snub cubical graph as depicted in Figure 12, but in a triangular perimeter. The vertex numbering is consistent with Table 1, Figure 5 and Figure 9. The apical 12-vertex subgraph (blue vertices) is inscribed within a basal 12-vertex subgraph (green vertices). Consider the two 12-vertex sub-graphs are representations of the two sets of 12 generators, selected (non-mixing) and deselected (mixing), in $S U(5)$. Let us consider the green vertices selected, and the blue vertices deselected. The number of coupling-edges connecting the two sub-graphs is minimized to twelve (coloured cheddar-orange). There are 24 blue edges connecting blue vertices and 24 green edges connecting green vertices.

In this Tribonacci-QC model dimensions are shared. A challenge is to discover the correspondence between the 12(2)-generators that are isomorphic to particle physics and to the degrees of freedom written in spherical codes that are isomorphic to dimensions of 24-spacetime, and its coarser manifestation, 4(6)-spacetime.

Figure 10 depicts the placement of the 12 Naturally selected (and deselected) generators of $S U(5)$ at the 4 vertices of the coarsened graph, i.e. the tetrahedral graph, representing the spherical tetrahedral spherical code ${ }_{\star}^{\star} C^{4(6)}$. In Figure 10 three Naturally selected generators are placed at each of the 4 tetrahedral vertices. An alternative placement is depicted in Figure 13, in which the 12 Naturally selected generators of $S U(5)$ are placed anti-clustered, enclosing the time-like apex, i.e. constituting the space-like base.

These alternative correspondences (Figure 10 versus Figure 13) are presented in this introduction of the Tribonacci-QC model to prompt further research, including by tropical geometricians and algebraists, into determining the best correspondences between the dimensions of spacetime and the dimensions of the 
Standard Model of particle physics. Furthermore, these two alternative correspondences are non-exclusive, further alternatives should be sought and contrasted.

Parenthetically, Borsten et al. [93] derive $E_{8}$ symmetry from 8 qutrits, which relates to the self-assembly of eight 3 -vertex codes into the 24 -vertex code of Tribonacci-QC (Figure 2 and Figure 4). The $E_{8}$ Lie group has applications in several definitions of $G_{s m}$.

In Section 2, above, I point out that a complementary path to map the 24 -vertex set snub cube to the 4(6)-vertex set tetrahedron is via the 24 -cell and the binary tetrahedral group of order 24 (2.11). The group of unit quaternions is the double cover of the $3 \mathrm{~d}$ rotation group. There are $12(2)=24$ unit quaternions that give rotational symmetries of the tetrahedron. These form the binary tetrahedral group. Unit quaternions thus link the tetrahedron to the number 24 and elliptic curves. The 24 unit quaternions in the binary tetrahedral group are also the Hurwitz integers of norm 1. The reader is directed to Braun's recent work on the 24-Cell and Calabi-Yau threefolds with Hodge numbers $(1,1)$ [94], Witten's seminal contributions, for example [95], those of Gross et al. [96] and to the works of Morrison and Vafa [97] [98] on compactifications on Calabi-Yau threefolds, which emphasise the fundamental relationship between those fields of research and the number 24 .

I note the most promising approaches to formalize the foundations of physics deeply incorporate the number 24 and we should seek even deeper understanding of this exceptional number, particularly as it relates to computational degrees of freedom. I propose this future exploration be done with reference to the 24-vertex snub cube spherical code and its parallelized 4(6)-vertex tetrahedron spherical code of Tribonacci-QC.

Finally, in this section, a recap of some profoundly simple mathematics related to the number 24 . The number 24 has more divisors (exactly eight) than any smaller number, making it a highly composite number which provides computational utility with dimensional parsimony. 24 is the $4 \mathrm{D}$ kissing number [99]. 24 is also the factorial of $4(24=4$ !) and the tesseract (the $4 \mathrm{D}$ analogue of the cube) has 24 two-dimensional faces, each of which is a 4 -sided square. The product of any 4 consecutive numbers is divisible by 24 and 24 is the number of ways to order 4 distinct items.

The 12(2)-generators (i.e. 12 mixing and 12 non-mixing) in Furey's work [90] on division algebras and $S U(5)$ ladder symmetries yields:

$$
2 \times 12=24
$$

Borsten et al. [93] derive $E_{8}$ symmetry from eight 3-level qutrits:

$$
3 \times 8=24
$$

Baez makes a simple but profound observation, that 4-fold and 6-fold lattices in the plane have the most symmetries and the product of their fold is given by:

$$
4 \times 6=24
$$

Similarly, deeply related to the Standard Model of particle physics, the special 
unitary group of degree $5, S U(5)$, is the Lie group of $5 \times 5$ unitary matrices with determinant 1 and can be represented:

$$
5 \times \overline{5}-1=24
$$

Finally, as we discuss in Section 4.1, the number of permutations of a signature code, of a certain third order recurrence sequence, the tribonacci sequence, drawn from the balanced ternary set $\{-1,0,1\}$ and which are not single valued is

$$
3^{3}-3=24
$$

\section{Snub Cube Characteristics}

In this section I discuss the characteristics of the snub cube, firstly and briefly in an introductory way. Then two sub-sections cover details pertaining to the fundamentally related tribonacci constant and tribonacci sequence (Section 4.1) and to the chirality and twist angle of the snub cube (Section 4.2).

The snub cube is an Archimedean solid polyhedron. Kepler studied it in his Harmonices Mundi of 1619 and he called it the cubus simus. I humbly suggest Kepler's intuitive search for a connection between the snub cube and cosmology is being continued in this present research, albeit building on four hundred years of scientific progress.

The snub cube has 24 vertices, 60 edges and 38 faces, 6 of which are squares and 32 of which are equilateral triangles. It can be represented as a spherical tiling and as a graph, as above. The symmetry group is octahedral, with order 24 , and it is thus sometimes called the snub cuboctahedron. It is chiral, having two enantiomorphs, levo and dextro.

The tribonacci constant, $T$, is implicit in the snub cube, specifically, the Cartesian coordinates for the vertices of a snub cube are all the even permutations of

$$
\left( \pm 1, \pm \frac{1}{T}, \pm T\right)
$$

with an even number of plus signs, together with all the odd permutations with an odd number of plus signs, and where $T \approx 1.8392867552 \cdots$ is the tribonacci constant (Section 4.1). The even permutations with an odd number of plus signs and the odd permutations with an even number of plus signs, gives the enantiomorph.

Its circumradius, $R$, is given by $[20]$

$$
R=\sqrt{\frac{3-T}{8-4 T}}
$$

and (4.1) and (4.2), together with the geometrical properties of spherical equilateral triangles, lead to (2.4).

\subsection{Tribonacci Constant and Sequence}

Whereas the well-known Fibonacci numbers start with two given terms, the lesser known tribonacci numbers [100] [101] [102] start with three given terms and 
each term thereafter is the sum of the preceding three. The first positive tribonacci numbers with a "signature code" $(0,0,1)$ are thus

$$
0,0,1,1,2,4,7,13,24,44,81, \cdots
$$

formalized as a sequence of integers $T_{n}$, where, here also illustrated with a signature code $(0,0,1)$,

$$
T_{0}=T_{1}=0, T_{2}=1, T_{n}=T_{n-1}+T_{n-2}+T_{n-3}
$$

The tribonacci constant, $T$, is the ratio toward which adjacent tribonacci numbers tend, which is also the real root of the polynomial

$$
T^{3}-T^{2}-T-1=0
$$

alternatively, written

$$
T^{3}=T^{2}+T+1
$$

and where $T$ is the real eigenvalue of the polynomial companion matrix, $M$, where

$$
M=\left(\begin{array}{lll}
0 & 1 & 0 \\
0 & 0 & 1 \\
1 & 1 & 1
\end{array}\right)
$$

and

$$
T=\frac{1+\sqrt[3]{19+3 \sqrt{33}}+\sqrt[3]{19-3 \sqrt{33}}}{3}
$$

The Tribonacci constant, $T$, can also be expressed as an infinite nested radical expression

$$
\frac{1}{T-1}=\sqrt[3]{\frac{1}{2}+\sqrt[3]{\frac{1}{2}+\sqrt[3]{\frac{1}{2}+\sqrt[3]{\frac{1}{2}+\sqrt[3]{\frac{1}{2}}+\cdots}}}}
$$

and the decimal approximation of the tribonacci constant is $T \approx 1.8392867552 \cdots$.

Let us now consider the effect of the signature code on the tribonacci sequence. Above, (4.1.1) and (4.1.2) pertain to the signature code $(0,0,1)$. In principle, the signature code could comprise any three numbers, including negative numbers and imaginary numbers. Here, we shall use $-1,0$ and 1 . Furthermore, let us envisage a balanced ternary, three-level quantum system, quantum trit or qutrit, with $3^{3}=27$ permutations of $-1,0$ and 1 , with three orthonormal basis states or vectors, denoted

$$
|i\rangle,|j\rangle,|k\rangle
$$

where

$$
i, j, k \in\{-1,0,1\}
$$

Whilst there are 27 permutations (Figure 14), if we apply a rule to exclude the three extrema qutrit signature codes which are single valued, i.e. where three basis states are all -1 , or all 0 , or all 1 , then there are 24 qualifying qutrit signature codes. The Natural proclivity of 24 returns to mind (3.11). 


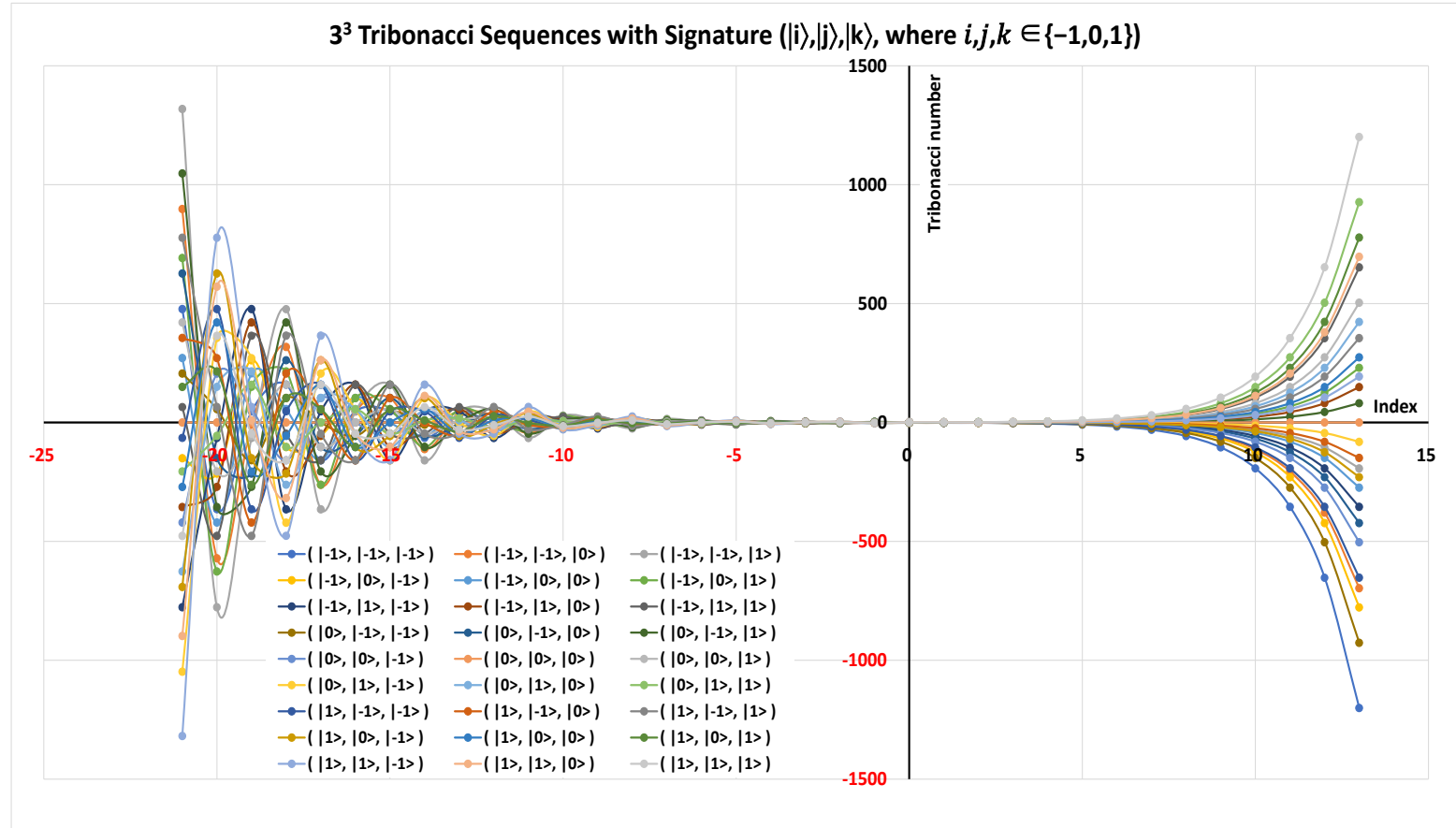

Figure 14. Plot of the remarkable evolution of the values (ordinate) of numbers in third order tribonacci sequences versus their indices (abscissa), for 27 different signature codes, $|i\rangle,|j\rangle,|k\rangle$, where $i, j, k \in\{-1,0,1\}$, corresponding to the zeroth, first and second index.

In Figure 14 we see how the permutations of the qutrit signature code (4.1.8) (4.1.9), corresponding to the zeroth, first and second index, affect the tribonacci sequences.

Let us consider this as an evolution from large negative indices (left) to increasingly large positive indices (right). We observe large and chaotic tribonacci numbers with large negative indices, which flip-flop between negative and positive values. This chaotic behaviour progressively dampens to the right, towards the signature code. The negative indices exhibit a double-sided decaying exponential signal. The signature code suppresses the chaotic oscillation, like a regulating gate.

Further to the right, through the gate (signature code), with increasing positive indices, the tribonacci numbers again become very large, but now the growth is smooth, exponentially rising and it is either positive or negative i.e. a single-sided (not double-sided) signal. The sign of the tribonacci sequence for positive indices depends on the numbers in the signature code. The signature code rectifies the sequence for positive indices.

I conjecture that a quantum computer with snub cubical graph architecture would exhibit behaviours related to the metric properties of the 24-vertex snub cube and thus to the tribonacci constant. Furthermore, I propose a balanced ternary, qutrit, deep learning, snub cubical quantum computer would suppress and harness primordial chaos and thence govern orderly cosmological expansion and the epistemic accumulation of advantageous knowledge, in the manner of the underlying tribonacci sequence evolution shown in Figure 14. Qutrit signature 
codes in Tribonacci-QC regulate and rectify chaos into orderly expansion.

See [23] for a discussion on tribonacci matrices and a new coding theory. Applications of Fibonacci numbers include computer algorithms such as the Fibonacci search technique and the Fibonacci heap data structure, and graphs called Fibonacci cubes used for interconnecting parallel and distributed systems. In Tribonacci-QC, cosmological and quantum mechanical information processing, based on fundamental tribonacci (rather than Fibonacci) sequences, are postulated to be Naturally selected.

The reader is also referred to the work of Cerda-Morales who formalizes tribonacci-type octonions and quaternions sequences and describes their properties [24] [25], which have profound occurrences in Nature and provides a link to octonion particle physics research [90].

Let us now consider this deep learning Tribonacci-QC as one in which $\mathrm{Na}$ ture's fundamental computations are 3-level qutrit operations. The universe, or Triuniverse [1], is an evolving system of qutrit quantum computations, with innate ternary quantum deep learning. Fundamental information processing is driven by Epistemic Drive, the Natural appetite for information selected for advantageous knowledge. Evolution of the Triuniverse occurs through the operation of quantum Darwinian and classical Darwinian deep learning processes. Darwin's universal 3-step evolutionary algorithm of Variation, Selection and Replication is claimed to be fundamental to Natural deep learning and knowledge growth across all scales; from the macroscopic cosmos, through mesoscopic Life, to sub-microscopic quantum physics.

As in Dirac's belt trick, two loops of this evolutionary algorithm return the identity, which relates to the $4 \pi$ identity rotation of a spinor. The two loops are here defined to be those of a directed trefoil torus knot (Figure 15 and Figure 16).

Figure 15 and Figure 16 illustrate the universal 3-step evolutionary algorithm of Variation, Selection and Replication. The captions of these figures guide the reader through the processing steps of the algorithm and explain its foundations on a 3-vertex directed graph which relates to a trefoil torus knot. The two-loop processing cycle, $C$, is an Eulerian cycle comprising subprocesses at its vertices: Variation, $\stackrel{z}{\Rightarrow} V \stackrel{w}{\Rightarrow}$ with input $z$ and output $w$, Selection, $\stackrel{w}{\Rightarrow} S \stackrel{x}{\Rightarrow}$ with input $w$ and output $x$ and Replication, $R \stackrel{y}{\Rightarrow} R$ with input $x$ and output $z$. The Replication subprocess (blue) is distinguished by its intrinsic replication sub-cycle, $y$.

The Darwinian Eulerian cycle of Variational-Selective-Replication, $C$, is defined as

$$
C=\{\cdots V \stackrel{w}{\Rightarrow} S \stackrel{x}{\Rightarrow} R \stackrel{y}{\Rightarrow} R \stackrel{z}{\Rightarrow} V \cdots\}
$$

From graph theory, the adjacency matrix, $L$, corresponding to this Eulerian cycle, $C$, is

$$
L=\left(\begin{array}{lll}
0 & 1 & 0 \\
0 & 0 & 1 \\
1 & 1 & 1
\end{array}\right)
$$



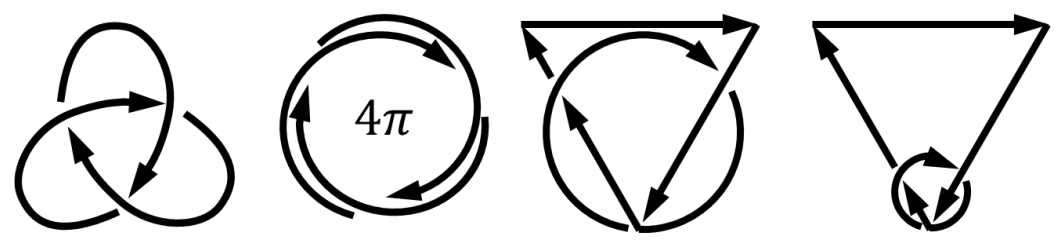

Figure 15. A directed trefoil torus knot is illustrated in four topologically equivalent configurations. From left to right, the first is the most familiar configuration of a right-handed trefoil torus knot. The second configuration emphasises its intrinsic $4 \pi$ identity rotation. The third configuration introduces three vertices and the fourth configuration displays an overhand knot sub-cycle around one vertex.

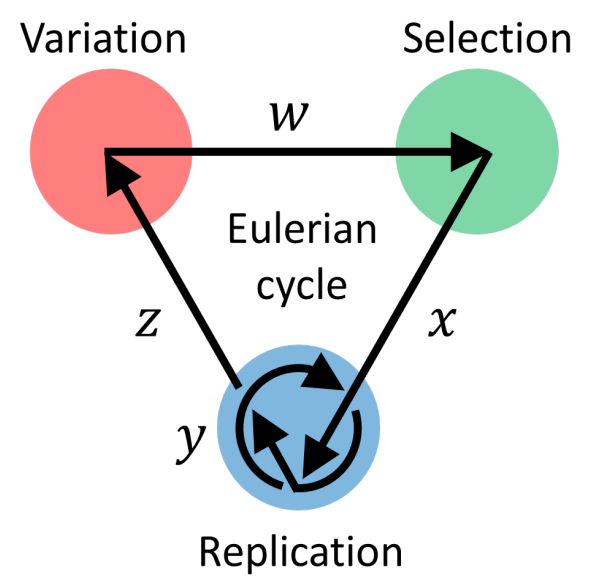

Figure 16. A diagram of a universal Darwinian, evolutionary, quantum deep learning, information processing cycle, $C$, represented by an Eulerian cycle through 3 vertices of a directed graph. The configuration of the directed graph is identical to that shown at the right-hand end of Figure 15. Each vertex represents a subprocess in the algorithm. From top left, clockwise, the first vertex represents the subprocess of Variation $\stackrel{z}{\Rightarrow} V \stackrel{w}{\Rightarrow}$ (red) and has input $z$ and output $w$. The second vertex represents the subprocess of Selection $\stackrel{w}{\Rightarrow} S \stackrel{x}{\Rightarrow}$ (green), with input $w$ and output $x$. The third vertex is the subprocess of Replication $R \stackrel{y}{\Rightarrow} R$ (blue), with input $x$ and output $z$. The Replication subprocess (blue) is distinguished by its intrinsic replication sub-cycle, $y$.

In this deep learning Tribonacci-QC model an equivalence is proposed, such that

$$
L \equiv M
$$

where $L$ is given by (4.1.11) and the polynomial companion matrix, $M$, is given by (4.1.5), and we recall the tribonacci constant, $T$, is the real eigenvalue of the polynomial companion matrix, $M$.

Such a proposed equivalence relates Darwin's universal, deep learning, algorithm of Variational-Selective-Replication to the tribonacci constant. See also Podani et al. [103] for an account of Darwin's model of a growing population of elephants as a tribonacci sequence.

In closing this subsection, let us consider other characteristics of matrices $M$ and $L$, specifically the eigenvalues: 


$$
\begin{gathered}
\lambda_{1}=T \approx 1.83929 \\
\lambda_{2}=\frac{1}{3}-\frac{1}{6}(1+i \sqrt{3}) \sqrt[3]{19-3 \sqrt{33}}-\frac{1}{6}(1-i \sqrt{3}) \sqrt[3]{19+3 \sqrt{33}} \\
\lambda_{2}=p_{1}+q_{1} i \approx-0.419643+0.606291 i \\
\lambda_{3}=\frac{1}{3}-\frac{1}{6}(1-i \sqrt{3}) \sqrt[3]{19-3 \sqrt{33}}-\frac{1}{6}(1+i \sqrt{3}) \sqrt[3]{19+3 \sqrt{33}} \\
\lambda_{3}=p_{2}+q_{2} i \approx-0.419643-0.606291 i
\end{gathered}
$$

where $p_{1}=p_{2}$ and is the

$$
\begin{aligned}
& \text { real root of } 4 x^{3}-4 x^{2}+1=\frac{1-T}{2} \approx-0.419643 \\
& p_{1}=p_{2}=\frac{1}{6}\left(2-\frac{4}{\sqrt[3]{19-3 \sqrt{33}}}-\sqrt[3]{19-3 \sqrt{33}}\right)
\end{aligned}
$$

and may be compared with (4.1.6).

We note from (4.1.18)

$$
\left|p_{1}\right|=\left|p_{2}\right|=\frac{T-1}{2} \approx 0.419643
$$

and we shall return to (4.1.20) in a proposed equivalent physical parameter in Section 7 (cosmographic distance ratio).

Furthermore, $q_{1}=-q_{2}$ and is the

$$
\text { real positive root of } 16 x^{6}-32 x^{4}+16 x^{2}+11 \approx 0.606291
$$

and eigenvectors of matrices $M(4.1 .5)$ and $L(4.1 .11)$ are:

$$
\begin{gathered}
v_{1}=\left(\frac{1}{T^{2}}, \frac{1}{T}, 1\right) \approx(0.295598,0.543689,1) \\
v_{2} \approx(-0.647799+1.72143 i,-0.771845-1.11514 i, 1) \\
v_{3} \approx(-0.647799-1.72143 i,-0.771845+1.11514 i, 1)
\end{gathered}
$$

\subsection{Chirality and Twist Angle}

Further important characteristics of the snub cube are its chirality and twist angle. Given the significance in this Tribonacci-QC model of the 24-vertex snub cubical optimal non-antipodal code and its isomorphism to 24-spacetime, we shall briefly discuss these characteristics. Their physical manifestations are discussed further in Sections 5, 6 and 8 .

Unlike most uniform polyhedral, the snub cube has two enantiomorphs (Figure 17), governed by their levo and dextro vertex configurations (4.1). In this Tribonacci-QC model, self-organization of optimal non-antipodal codes, as depicted in Figure 2, attains a 24-vertex configuration through combining eight 3-vertex codes on a noisy spherical horizon. That noise causes the dextro and levo enantiomorphs of the snub cube to be equiprobable outcomes of the quantum self-organization process. 

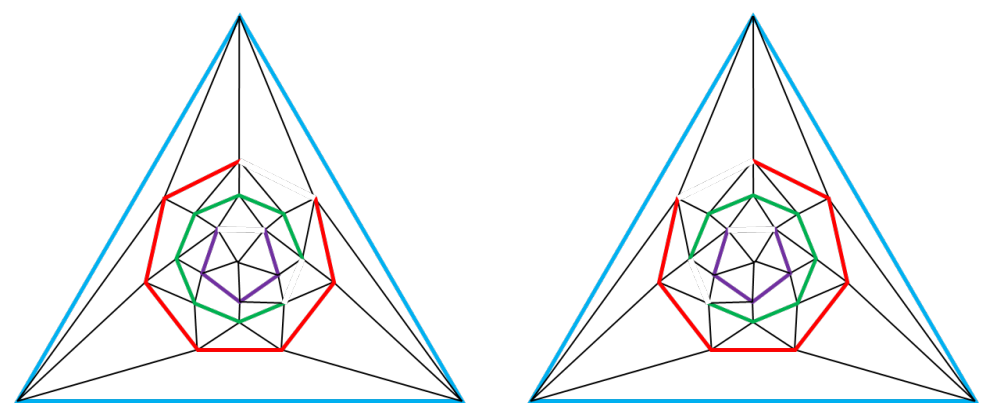

Figure 17. Snub cubical graph (from Figure 3), in a triangular perimeter (light blue), showing its two enantiomorphs, governed by their levo and dextro vertex configurations (4.1).

In this Tribonacci-QC model, the random outcome of one enantiomorph over the other sets up isomorphic 24-spacetime to be either matter-dominated or antimatter-dominated. The chirality of the snub cube underpins the observed matter-antimatter asymmetry, which in our present cosmological enantiomorph is in favour of matter.

The snubification twist angle, $\theta$, is given by [20]:

$$
\theta=\arccos (\sqrt{T / 2}) \approx 0.28741
$$

In this Tribonacci- $Q C$ model the twist angle divided by the spinor identity rotation, $4 \pi$, (Figure 15 ) defines a ratio, $\beta$, where

$$
\beta=\theta / 4 \pi=\arccos (\sqrt{T / 2}) / 4 \pi \approx 0.02287
$$

We shall return to this ratio, $\beta$, in Section 6, where a further relationship with matter is proposed (physical baryon density parameter).

I postulate that the snub cubical (and snub cubical coarsened into tetrahedral) optimal non-antipodal codes of Tribonacci- $Q C$ are isomorphic to corresponding chiral graphs which are stressed-graphs. The graph-stress partitions into shear graph-stress, ${ }_{4(6)}^{24} \tau$, and normal graph-stress, ${ }_{4(6)}^{24} \sigma$. The shear graph-stress in these chiral graphs relates to its chirality, quantified by twist. The normal graph-stress in these chiral graphs relates to the normal edge network. Both the shear and normal graph-stress, being intrinsic to their foundational snub cubical graph, relate to the tribonacci constant, $T$. I further postulate that the shear graph-stress component relates to matter, whereas the normal graph-stress component relates to spacetime and that this partitioning is manifest through a very well-known physical equivalence.

Consider General Relativity in 4-spacetime, as expressed in Einstein's effective tensor equivalence

$$
G_{\mu \nu}=\kappa \mathcal{T}_{\mu \nu}
$$

The left-hand side, to coin Wheeler's phraseology, relates to spacetime, which tells matter how to move, and the right-hand side refers to matter, that tells spacetime how to curve. 
In Tribonacci- $Q C$, foundational quantum information processing involves an error-correcting tribonacci coding-decoding procedure [23] that establishes dynamic equivalence in information-energy exchanges that are coded and decoded across a parallel computing partition ( $\|$ ). I propose the left-hand side of that partition computes the normal graph-stress component, ${ }_{4(6)}^{24} \sigma$, of the snub cubical graph, whereas the right-hand side computes the shear graph-stress component, ${ }_{4(6)}^{24} \tau$. Dynamic equivalence in information-energy exchanges is attained in quantized computational steps.

$$
{ }_{4(6)}^{24} \sigma \| \xi_{4(6)}^{24} \tau
$$

I conjecture that the tensor equivalence for General Relativity (4.2.3) corresponds to quantum computational partitioning represented by graph-stress in snub cubical (and snub cubical coarsened into tetrahedral) optimal non-antipodal codes, in Tribonacci-QC (4.2.4), which yields a quantum gravity correspondence, $Q G$, as follows

$$
Q G\left\{\begin{array}{l}
G_{\mu \nu}=\kappa \mathcal{T}_{\mu v} \\
{ }_{4(6)} \sigma \| \xi_{4(6)}^{24} \tau
\end{array}\right.
$$

Decompositions of the snub cubical graph adjacency matrix:

$\left[\begin{array}{llllllllllllllllllllllll}0 & 1 & 1 & 1 & 1 & 1 & 0 & 0 & 0 & 0 & 0 & 0 & 0 & 0 & 0 & 0 & 0 & 0 & 0 & 0 & 0 & 0 & 0 & 0 \\ 1 & 0 & 0 & 0 & 0 & 1 & 0 & 0 & 0 & 0 & 0 & 0 & 0 & 0 & 0 & 0 & 0 & 0 & 0 & 1 & 0 & 1 & 0 & 1 \\ 1 & 0 & 0 & 0 & 1 & 0 & 0 & 0 & 0 & 0 & 0 & 0 & 0 & 0 & 0 & 0 & 0 & 0 & 1 & 0 & 0 & 0 & 1 & 1 \\ 1 & 0 & 0 & 0 & 1 & 1 & 0 & 0 & 0 & 0 & 0 & 0 & 0 & 0 & 0 & 0 & 0 & 1 & 0 & 0 & 1 & 0 & 0 & 0 \\ 1 & 0 & 1 & 1 & 0 & 0 & 0 & 0 & 0 & 0 & 0 & 0 & 0 & 0 & 0 & 0 & 0 & 0 & 0 & 0 & 1 & 0 & 1 & 0 \\ 1 & 1 & 0 & 1 & 0 & 0 & 0 & 0 & 0 & 0 & 0 & 0 & 0 & 0 & 0 & 0 & 1 & 0 & 0 & 0 & 0 & 1 & 0 & 0 \\ 0 & 0 & 0 & 0 & 0 & 0 & 0 & 1 & 1 & 0 & 0 & 0 & 0 & 0 & 0 & 0 & 0 & 0 & 1 & 1 & 0 & 0 & 0 & 1 \\ 0 & 0 & 0 & 0 & 0 & 0 & 1 & 0 & 1 & 0 & 0 & 0 & 0 & 0 & 1 & 1 & 0 & 0 & 0 & 1 & 0 & 0 & 0 & 0 \\ 0 & 0 & 0 & 0 & 0 & 0 & 1 & 1 & 0 & 0 & 0 & 0 & 1 & 1 & 0 & 1 & 0 & 0 & 0 & 0 & 0 & 0 & 0 & 0 \\ 0 & 0 & 0 & 0 & 0 & 0 & 0 & 0 & 0 & 0 & 1 & 0 & 1 & 1 & 0 & 0 & 0 & 0 & 0 & 0 & 1 & 0 & 1 & 0 \\ 0 & 0 & 0 & 0 & 0 & 0 & 0 & 0 & 0 & 1 & 0 & 1 & 0 & 1 & 0 & 0 & 0 & 1 & 0 & 0 & 1 & 0 & 0 & 0 \\ 0 & 0 & 0 & 0 & 0 & 0 & 0 & 0 & 0 & 0 & 1 & 0 & 0 & 0 & 1 & 1 & 1 & 1 & 0 & 0 & 0 & 0 & 0 & 0 \\ 0 & 0 & 0 & 0 & 0 & 0 & 0 & 0 & 1 & 1 & 0 & 0 & 0 & 1 & 0 & 0 & 0 & 0 & 1 & 0 & 0 & 0 & 1 & 0 \\ 0 & 0 & 0 & 0 & 0 & 0 & 0 & 0 & 1 & 1 & 1 & 0 & 1 & 0 & 0 & 1 & 0 & 0 & 0 & 0 & 0 & 0 & 0 & 0 \\ 0 & 0 & 0 & 0 & 0 & 0 & 0 & 1 & 0 & 0 & 0 & 1 & 0 & 0 & 0 & 1 & 1 & 0 & 0 & 0 & 0 & 1 & 0 & 0 \\ 0 & 0 & 0 & 0 & 0 & 0 & 0 & 1 & 1 & 0 & 0 & 1 & 0 & 1 & 1 & 0 & 0 & 0 & 0 & 0 & 0 & 0 & 0 & 0 \\ 0 & 0 & 0 & 0 & 0 & 1 & 0 & 0 & 0 & 0 & 0 & 1 & 0 & 0 & 1 & 0 & 0 & 1 & 0 & 0 & 0 & 1 & 0 & 0 \\ 0 & 0 & 0 & 1 & 0 & 0 & 0 & 0 & 0 & 0 & 1 & 1 & 0 & 0 & 0 & 0 & 1 & 0 & 0 & 0 & 1 & 0 & 0 & 0 \\ 0 & 0 & 1 & 0 & 0 & 0 & 1 & 0 & 0 & 0 & 0 & 0 & 1 & 0 & 0 & 0 & 0 & 0 & 0 & 0 & 0 & 0 & 1 & 1 \\ 0 & 1 & 0 & 0 & 0 & 0 & 1 & 1 & 0 & 0 & 0 & 0 & 0 & 0 & 0 & 0 & 0 & 0 & 0 & 0 & 0 & 1 & 0 & 1 \\ 0 & 0 & 0 & 1 & 1 & 0 & 0 & 0 & 0 & 1 & 1 & 0 & 0 & 0 & 0 & 0 & 0 & 1 & 0 & 0 & 0 & 0 & 0 & 0 \\ 0 & 1 & 0 & 0 & 0 & 1 & 0 & 0 & 0 & 0 & 0 & 0 & 0 & 0 & 1 & 0 & 1 & 0 & 0 & 1 & 0 & 0 & 0 & 0 \\ 0 & 0 & 1 & 0 & 1 & 0 & 0 & 0 & 0 & 1 & 0 & 0 & 1 & 0 & 0 & 0 & 0 & 0 & 1 & 0 & 0 & 0 & 0 & 0 \\ 0 & 1 & 1 & 0 & 0 & 0 & 1 & 0 & 0 & 0 & 0 & 0 & 0 & 0 & 0 & 0 & 0 & 0 & 1 & 1 & 0 & 0 & 0 & 0\end{array}\right]$


and decompositions of the corresponding 24-vertex set (4.1) are conjectured to yield rich future insights into the quantum gravity correspondence, $Q G$, (4.2.5), and models of particle physics and cosmology.

\section{Cosmological Scale Factor Evolution}

The preceding Sections 1 to 4 introduce the foundations of Tribonacci-QC and provide context to now define the cosmological scale factor evolution, $a(t)$. Referring to (4.2.3) (4.2.4) (4.2.5) we now contextualize the formalizations in this Section 5 within the normal graph-stress component, ${ }_{4(6)}^{24} \sigma$, of the snub cubical graph.

From the following formalized cosmological scale factor evolution, equations are then derived for the Hubble parameter (Section 5.1) and deceleration-acceleration transition redshift (Section 5.2).

In Tribonacci- $Q C$, the transition from dominantly 24 -spacetime to dominantly 4(6)-spacetime (Section 2, Figure 2) necessitates, by definition, an abrupt change in $a(t)$ at $t=0$, with an equation for $a(t)$ when $t<0$, an equation for $a(t)$ when $t>0$ and the two equations Naturally splicing continuously at transition time $t=0$. Furthermore, a characteristic difference between the two equations is that during time $t<0$ (in 24-spacetime) the pace of time is 6 -fold faster than in parallelized 4(6)-spacetime.

Let us first focus on formalizing the cosmological scale factor evolution, $a(t)$, when $t>0$, i.e. in 4(6)-spacetime, because this is our spatio-temporal framework for present cosmological observations. We shall then modify its equation for time $t<0$ (in 24-spacetime), when the pace of time was 6-fold faster.

The equation, in Tribonacci-QC, for cosmological scale factor evolution, $a(t)$, when $t>0$, is defined:

$$
a(t)=3^{t^{T / 3}-1}
$$

and when $t<0$, the cosmological scale factor evolution is defined in 6 -fold faster time:

$$
a(t)=3^{(6 t)^{T / 3}-1}
$$

In Figure 18, below, we see a plot of (5.1) and (5.2), for real (blue) and imaginary (orange) values and is Naturally smoothly spliced through the transition event at time $t=0$.

The ordinate intercept in Figure 18 (5.1) (5.2) for real values (blue) $\operatorname{Re}[a(t)]$ is:

$$
a(t)=\frac{1}{3} \text {, when } t=0
$$

A standard equation relates cosmological scale factor $a(t)$ and cosmological redshift $z$

$$
a(t)=\frac{1}{1+z}
$$




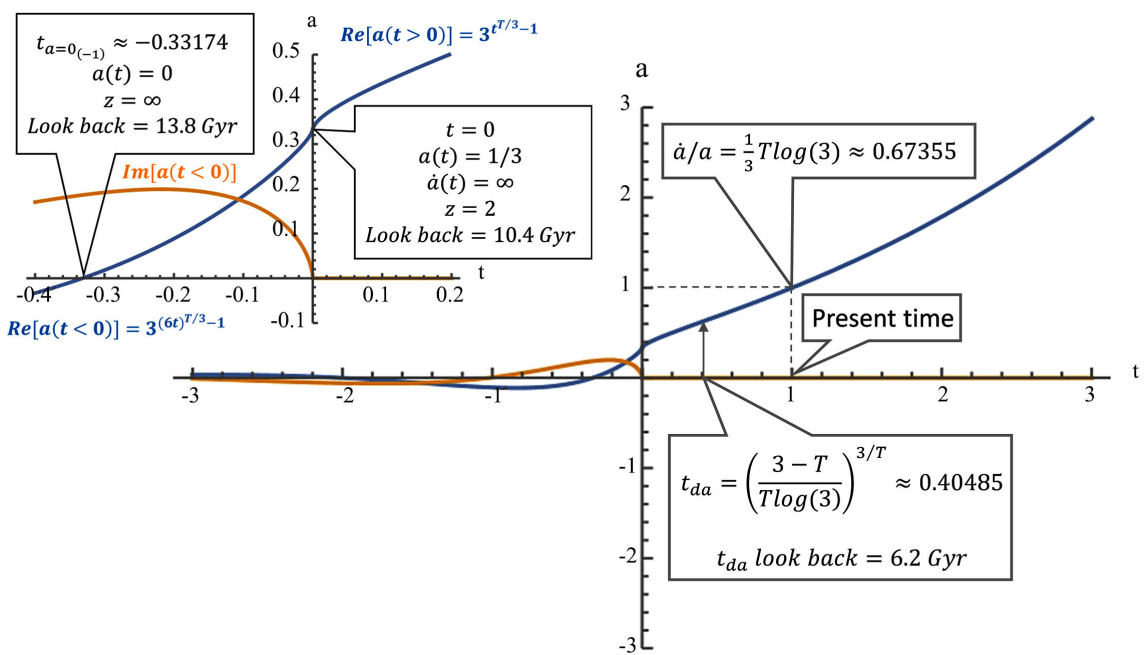

Figure 18. Plot with zoomed-in and annotated plot inset (top left) and further annotations (right). Cosmological scale factor $a(t)$ (ordinate) evolution in a plot of equations (5.1) and (5.2), for real values (blue) $\operatorname{Re}[a(t)]$ and imaginary values (orange) $\operatorname{Im}[a(t)]$ and Naturally smoothly spliced through $t=0$. Time $t$ (abscissa) is plotted from values -3 to 3 where $t=0$ is the time of transition from dominantly 24-spacetime $(t<0)$ to dominantly $4(6)$-spacetime $(t>0)$. Time $t=1$ is the present time, at which time, $a(t)=1$.

Substituting (5.3) in (5.4) gives

$$
z=2 \text {, when } t=0
$$

This result (5.5) is a novel and distinguishing characteristic of Tribonacci-QC and relates to the redshift $z=2$ when dominantly 24-spacetime transitions into dominantly 4(6)-spacetime. In Section 8.3 the significance of $z=2$ as a fundamental marker redshift in astronomical observations is discussed.

The real valued $a(t)$ abscissa intercept in Figure 18 (5.2), nearest $t=0$, in negative model time, corresponds to the time of last zero cosmological scale, when $a(t)=0$ :

$$
t_{a=0_{(-1)}} \approx-0.33174
$$

Let us now convert model time to look back time in billions of years (Gyr). Using an astronomical timescale [40], anchored on a cosmological age of 10.4 Gyr at redshift $z=2$, we thus calibrate

$$
t_{a=0(-1)} \approx a b s|-0.33174-1| \times 10.4 \approx 13.8 \mathrm{Gyr}
$$

The time of last zero cosmological scale (5.7) matches the Planck 2018 estimate [104] for the age of the observable universe.

The abscissa intercept in Figure 18 (5.2), second nearest to $t=0$, in negative model time, corresponding to the $2^{\text {nd }}$ last time of zero cosmological scale.

$$
t_{a=0_{(-2)}} \approx-1.99074
$$

By the same calibration, anchored on a cosmological age of 10.4 Gyr at red- 
shift $z=2$, we thus astronomically calibrate the $2^{\text {nd }}$ last time of zero cosmological scale as occurring at cosmological time:

$$
t_{a=0(-2)} \approx a b s|-1.99074-1| \times 10.4 \approx 31 \mathrm{Gyr}
$$

As a series, in negative model time, the real valued abscissa intercepts with zero cosmological scale factor (5.2) are:

$$
\begin{aligned}
t_{a=0_{(-1 \mathrm{to}-10 .)} \approx} & -0.33174,-1.99074,-4.58000,-7.92884,-11.94626, \\
& -16.57218,-21.76279,-27.48413,-33.70878,-40.41394, \cdots
\end{aligned}
$$

which corresponds to the following cosmological ages anchored on $10.4 \mathrm{Gyr}$ at redshift $z=2$

$$
\begin{aligned}
t_{a=0_{(-10-10 \cdots)}} \approx & 13.8 \mathrm{Gyr}, 31 \mathrm{Gyr}, 58 \mathrm{Gyr}, 93 \mathrm{Gyr}, 135 \mathrm{Gyr}, \\
& 183 \mathrm{Gyr}, 237 \mathrm{Gyr}, 296 \mathrm{Gyr}, 361 \mathrm{Gyr}, 430 \mathrm{Gyr}, \cdots
\end{aligned}
$$

In Tribonacci-QC, the cosmological scale factor evolution $a(t),(5.1)(5.2)$, is an Ostwald coarsening with the cube root of time [105] [106], scaled by the tribonacci constant ( $T \approx 1.83929$ ) metric property of the snub cubical 24-vertex and parallelized 4(6)-vertex spherical codes. Tribonacci-QC is a quantum foundational model founded on the integer base of quantum ternary computing. The integer base and denominator of the Ostwald coarsening exponent in equations (5.1) and (5.2) is 3, which has integer lowest radix economy, i.e. $\lceil\mathrm{e}\rceil=3,[1]$ [107]. The -1 in the exponent (5.1) (5.2) simply sets $a(t)=1$ at present time $t \rightarrow 1$, as a relevant frame of reference.

In Tribonacci-QC, Ostwald coarsening of optimal non-antipodal spherical codes broadens the bandwidth of quantum information processing on the spherical horizon. During coarsening, from the 24-vertex set to the parallelized 4(6)-vertex set, the average signal coarseness increases with the third root of time (as is characteristic of Ostwald coarsening), scaled by the tribonacci constant, $t^{T / 3}$. The concept of an evolving average coarseness is important.

It is an average signal coarseness related to two coexisting metastable states, 24-vertex and 4(6)-vertex, which spontaneously transition from dominantly 24 -vertex, to dominantly 4(6)-vertex states. In the physical universe, mixed states dominated by the 24-vertex state transition (at $t=0$ ) into mixed states dominated by the 4(6)-vertex state. In Tribonacci- $Q C$, the isomorphic dimensionality of 24-spacetime persists into times when $t>0$, including the present time, $t \rightarrow 1$, where it is proposed to manifest in at least two remnant configurations.

One of these remnant configurations of the 24-vertex state persists as the pervasive 12(2)-dimensional quantum physics realm (Section 3), microscopically and quantum graphically circumscribed within the spacetime fabric of the parallelised 4(6)-vertex state. The other of these remnant configurations of the 24-vertex state persists as Planck stars [45] at the cores of black holes.

At the coarsening transition, antecedent, quantum astrophysical structures created in dimensions of 24-spacetime metamorphose into their familiar 4(6)- 
spacetime forms e.g. a 24-dimensional galaxy metamorphoses into a familiar 4(6)-dimensional galaxy. It is the same galaxy but is now manifest (conserving matter and energy) in fewer and parallelized degrees of freedom. Section 8 , on astronomical observations, expands on these themes.

Also recall Figure 13 which may be interpreted as a quantum mechanical configuration of a Planck star at the core of a black hole, where the circumscribed, apical, time-like vertices correspond to quantum mechanics concealed within the black hole (Section 8.2, below).

Figure 19, shows the evolution (5.2) of the real (blue) and imaginary (orange) values of $a(t)$ in dominantly 24 -spacetime, during negative model time $(t<0)$.

The real cosmological scale factor (blue), $\operatorname{Re}[a(t<0)]$, and imaginary cosmological scale factor (orange), $\operatorname{Im}[a(t<0)]$, evolve (5.2) in negative model time, from $t \rightarrow-\infty$ to $t=0$, as positive and negative valued scale factor oscillations with $a(t)$ amplitude increasing towards $t=0$. Positive valued scale factors project outside the optimal non-antipodal codes'spherical horizon, whereas negative scale factors project inside that spherical horizon.

Consider these two functions, $\operatorname{Re}[a(t<0)], \operatorname{Im}[a(t<0)]$, alternatively written as coupled differential equations, then their potential to represent an autocatalytic oscillating reaction [108] [109] could be studied. Alternatively, we may consider these two functions representing an entanglement of two energy-matter phases self-pumping in an epistemically-driven cavity bounded by the spherical horizon of the optimal non-antipodal codes, ${ }_{\leftrightarrow}^{\star} C \subset S^{2}$.

Pending such reformalizations, the real function $\operatorname{Re}[a(t<0)]$ (blue) is postulated to relate to matter, whereas the imaginary function $\operatorname{Im}[a(t<0)]$ (orange) relates to antimatter, and while the proposed autocatalytic oscillating reaction consumes all imaginary antimatter during negative time, from $t \rightarrow-\infty$ to $t=0$ (Figure 19), only real matter persists into positive time $t>0$ (Figure $18)$.

It is also informative to consider Figure 20 which shows a parametric plot of equation (5.2), when time $t<0$, during the evolution of dominantly 24-spacetime. The imaginary values of $a(t)$ are represented on the ordinate. The real values of $a(t)$ are represented on the abscissa. The origin $(0,0)$ of this plot represents the origin of time, when $t \rightarrow-\infty$.

Tribonacci-QC postulates that cosmological scale $a(t) \rightarrow 0$ as $t \rightarrow-\infty$, for both real and imaginary values. The arrow of time advances clockwise from the origin and the right-hand end of the spiral curve intersects the abscissa at $a(t)=1 / 3, \quad z=2$, when $t=0,(5.3)$ (5.5). The parametric plot shows that infinite negative time is an attracting fixed point of the reverse time evolution.

\subsection{Hubble Parameter}

The dimensionless Hubble parameter, $h$, has a standard definition:

$$
h=\frac{\dot{a}}{a}
$$




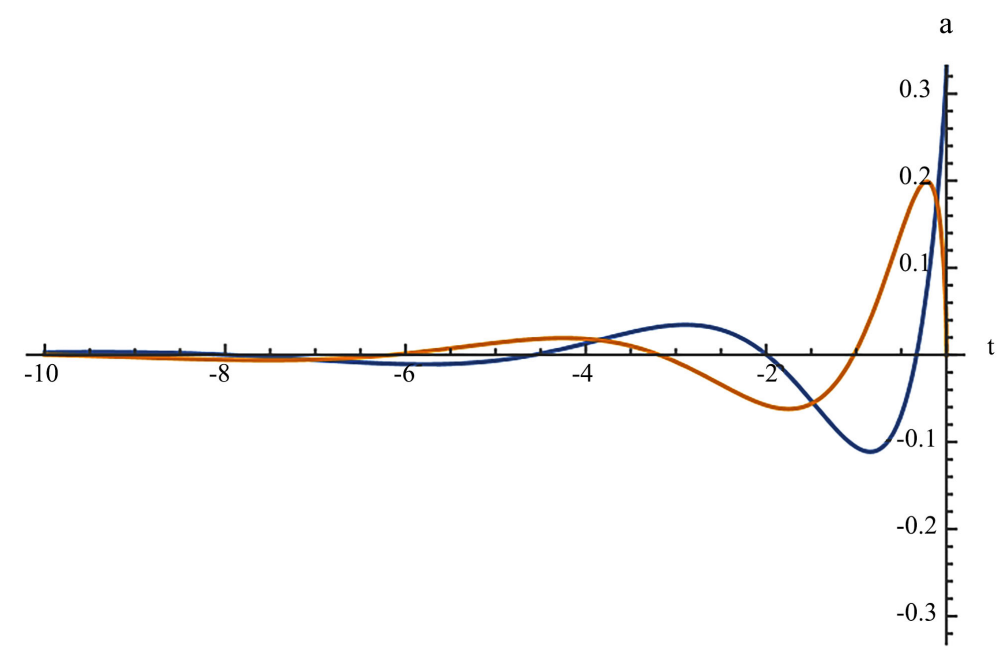

Figure 19. The real cosmological scale factor (blue), $\operatorname{Re}[a(t<0)]$, and imaginary cosmological scale factor (orange), $\operatorname{Im}[a(t<0)]$, evolve (5.2) in negative time, from $t \rightarrow-\infty$ to $t=0$, as positive and negative valued scale factor oscillations with $a(t)$ amplitude increasing towards $t=0$.

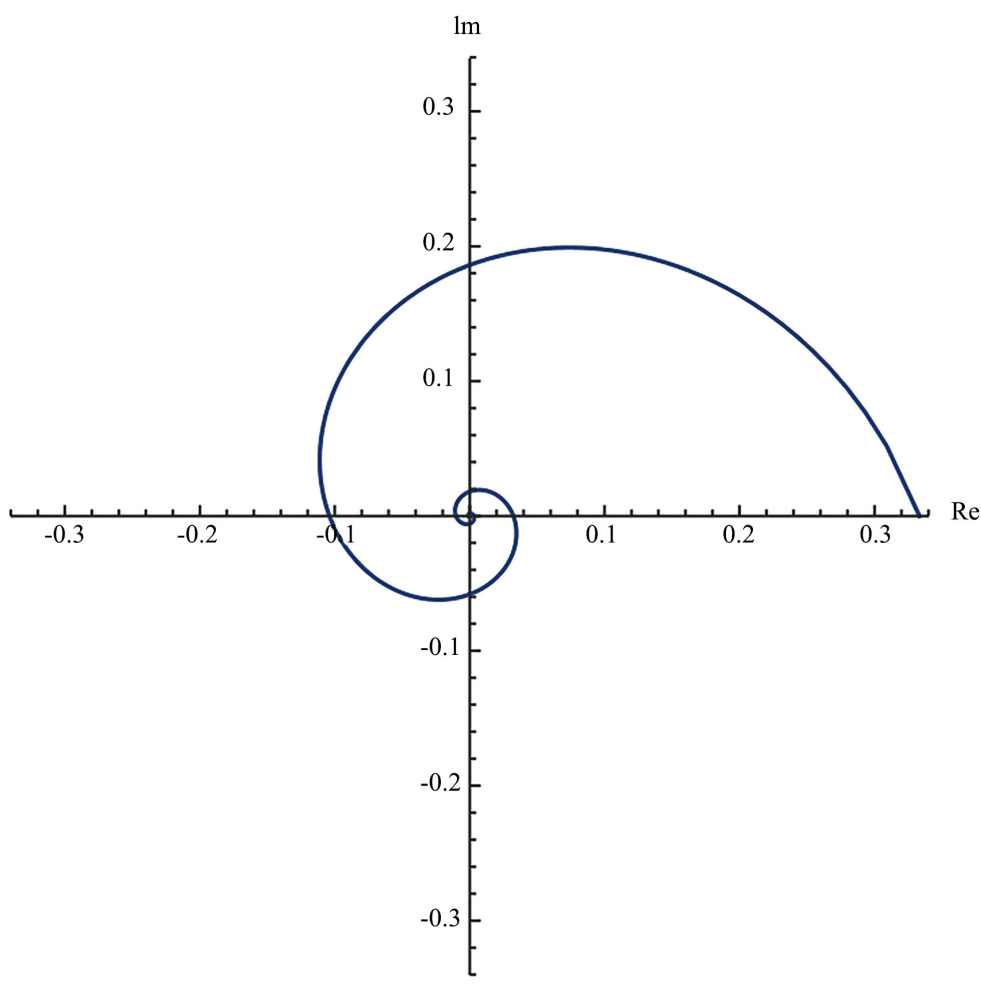

Figure 20. A parametric plot of Equation (5.2), when time $t<0$, during the evolution of dominantly 24-spacetime. The imaginary values of $a(t)$ are represented on the ordinate. The real values of $a(t)$ are represented on the abscissa. The origin $(0,0)$ of this plot represents the origin of time, when $t \rightarrow-\infty$.

Accordingly, we now study the evolution of Tribonacci-QC in positive time $t>0$ and substitute (5.1) into (5.12) to get: 


$$
h=\frac{1}{3} T \log (3) t^{T / 3-1}
$$

At present time, $t \rightarrow 1$, the Hubble parameter $h_{t \rightarrow 1}$ is:

$$
H_{0} / 100 \equiv h_{t \rightarrow 1} \equiv \dot{a} / a=\frac{1}{3} T \log (3) \approx 0.67355
$$

where $H_{0}$ is the Hubble "constant" and in Tribonacci-QC has value:

$$
H_{0} \approx 67.355 \mathrm{~km} \cdot \mathrm{s}^{-1} \cdot \mathrm{Mpc}^{-1}
$$

which is close to the Planck 2018 estimate [104].

However, there is a significant tension in modern cosmology. The value of $H_{0}$ in (5.15) differs from that derived by the distance ladder [110].

Let us consider the fractional quantum Hall effect (FQHE) which is a property of a collective state with fractional quantum statistics. For example, Pan et al. [111] report a fractional quantum Hall effect at Landau level filling $v=4 / 11$. Let us take their experimental evidence to a more general level, where I postulate that fractional quantum effects also occur in quantum cosmology on a very large scale through deep spacetime.

Revisiting this Tribonacci-QC model's Ostwald coarsening (particularly the denominator of the Ostwald coarsening exponent in Equations (5.1) and (5.2)), within such a cosmological fractional quantum paradigm, then we may consider fractional quantum plateaus developing near $\lceil\mathrm{e}\rceil=3$, which has integer lowest radix economy [1] [107]. Specifically, let us search for economic fractional quantum plateaus between $\mathrm{e} \approx 2.71828 \cdots$ and 3 . Prominent in this search, and close to the most economic radix, e, is the simple fraction $11 / 4=2.75$, which is the reciprocal of that reported by Pan et al. Experimenting with this thought, we now modify the Ostwald coarsening exponent in Tribonacci- $Q C$ from the $1 / 3$ root of time, to the near valued 4/11 [111] root of time, to discover:

$$
\frac{\dot{a}}{a}=h=\frac{4}{11} T \log (3) \approx 0.73479
$$

From (5.16) we determine

$$
H_{0} \approx 73.479 \mathrm{~km} \cdot \mathrm{s}^{-1} \cdot \mathrm{Mpc}^{-1}
$$

which is close to the value of the Hubble "constant" measured using the distance ladder approach [110].

I propose that Planck 2018 is measuring the effects of Ostwald coarsening in Tribonacci-QC where the denominator of the Ostwald coarsening exponent in Equations (5.1) and (5.2) has integer value $\lceil\mathrm{e}\rceil=3$, whereas in distance ladder methods it has fractional quantum value $\lceil\mathrm{e}\rceil=11 / 4$. The Wheelerian participatory observers' choices viz a viz configuration of instruments and particularly the direction of data processing (distance ladder versus inverse distance ladder, $\mathrm{CMB}$ and $\Lambda \mathrm{CDM}$ ) influence the quantum cosmological measurement, see Section 8.3.7. It would appear the direction of measurement in deep cosmological spacetime reveals a noncommutative characteristic of our expanding quantum computational universe. Spacetime coarsening in Tribonacci-QC, from 24 to 4(6) 
degrees of freedom is, I claim, noncommutative and the tension in the values of $H_{0}$, (5.15) versus (5.17), is due to the direction of our measurements, i.e. from the $\mathrm{CMB}$ to the observer (5.15) versus from the observer to high redshift supernovae (5.17).

It is noted that other simple fractions exist between $\mathrm{e} \approx 2.71828 \cdots$ and 3 which are closer to $e$, including 30/11, 41/15, 49/18 .. 1360/500 .. 67,958/25,000 ... but if we limit the numerator and denominator to computationally efficient values $\leq 24$, i.e. limited within the intrinsic degrees of freedom in Tribonacci-QC, then $\ldots$ only $11 / 4(=22 / 8)$ qualifies. That is, we discover $11 / 4$ is the exceptional simple fraction for efficient radix economy within Tribonacci-QC.

I also propose that other fractional quantum values related to new phenomena in quantum physics are expected in the neighbourhood of e, such as $14 / 5$ and including those slightly $<e$, such as $19 / 7,8 / 3,21 / 8,13 / 5$ and their reciprocals $5 / 14$, $7 / 19,3 / 8$ [111], 8/21 and 5/13 [111]. Fractional quantum phenomena in the neighbourhood of e are proposed to constitute evidence of quantum computational radix economy in the deep learning quantum information processing of Nature [1] [107].

\subsection{Deceleration-Acceleration Transition Redshift}

The deceleration-acceleration transition occurs at model time $t_{d a}$ when $\ddot{a}=0$. The second derivative of (5.1) when equal to zero and when $t_{d a}>0$ and real is given by:

$$
\begin{gathered}
\ddot{a}=T-3+\log (3) T t_{d a}^{T / 3}=0 \\
t_{d a}=\left(\frac{3-T}{T \log (3)}\right)^{3 / T} \approx 0.40485
\end{gathered}
$$

Adopting the model time calibration in (5.7), we calculate that $t_{d a}$, look back time $\approx 6.2$ Gyr ago. Substituting (5.19) in (5.1) when $t>0$ gives:

$$
a\left(t_{d a}\right)=\frac{1}{3} \mathrm{e}^{3 / T-1} \approx 0.62654
$$

Rewriting (5.20) in terms of cosmological redshift $z_{d a}$ using (5.4):

$$
z_{d a}=3 \mathrm{e}^{1-3 / T}-1 \approx 0.59607
$$

which compares well with supernovae observations [41] [42].

\section{Matter Density Parameters}

Referring to (4.2.5) and the above discussion in Section 4 on a postulated quantum gravity correspondence in Tribonacci- $Q C$, we contextualize the spacetime scale factor evolution formalizations in Section 5 (preceding) within the normal graph-stress component, ${ }_{4(6)}^{24} \sigma$, of the snub cubical graph.

Whereas, in this Section 6 we consider the shear graph-stress component, ${ }_{4(6)}^{24} \tau$, of the snub cubical graph, which I claim relates to the matter side of Einstein's spacetime-matter tensor equivalence (4.2.5). 
The physical baryon density parameter, $\Omega_{b} h^{2}$, is deeply fundamental to cosmology, and I commence this section with its definition in Tribonacci-QC. Returning to the 24-vertex snub cube, a fundamental shear graph-stress parameter is the snubification twist angle, $\theta,[20]$ which is formalized in (4.2.1).

Referring to Dirac's belt trick (Figure 15) and a $4 \pi$ identity rotation, in Tribonacci- $Q C$ the twist angle, $\theta$, divided by $4 \pi$ defines a ratio, $\beta$, (4.2.2). I postulate this ratio, $\beta$, relates fundamentally to the shear graph-stress component, ${ }_{4(6)}^{24} \tau,(4.2 .5)$ and is equivalent to the physical baryon density parameter, $\Omega_{b} h^{2}$, as follows:

$$
\Omega_{b} h^{2}=\beta=\theta / 4 \pi=\arccos (\sqrt{T / 2}) / 4 \pi \approx 0.02287
$$

Due to the 6-fold parallelization, from 24-spacetime to 4(6)-spacetime (Figure 2 ), the matter density parameter, $\Omega_{m}$, in Tribonacci- $Q C$ is 6 -fold heavier than the baryon density parameter, $\Omega_{b}$. Inserting the formalization of the Hubble parameter $h_{t \rightarrow 1}$ (5.14) in (6.1) gives:

$$
\Omega_{b}=\arccos (\sqrt{T / 2}) / 4 \pi\left(\frac{1}{3} T \log (3)\right)^{2} \approx 0.50414
$$

which is reconcilable with standard cosmic nucleosynthesis and the matter density parameter, $\Omega_{m}$, is:

$$
\Omega_{m}=6 \Omega_{b}=27 \arccos (\sqrt{T / 2}) / 2 \pi \log ^{2}(3) T^{2} \approx 0.30248
$$

which is close to the Planck 2018 result [104].

\section{Cosmographic Distance Ratio}

It is interesting to note the recent work of Miyatake et al. [43] on a measurement of the cosmographic distance ratio, $r$, with galaxy and cosmic microwave background lensing, where

$$
r=\gamma_{t}^{o} / \gamma_{t}^{c}
$$

and where $\gamma_{t}^{o}$ and $\gamma_{t}^{c}$ are the optical and cosmic microwave background tangential shear. Their measurement of $r$ leads me to propose another equivalence in Tribonacci-QC:

$$
\left|p_{1}\right|=\left|p_{2}\right|=r=\frac{\gamma_{t}^{o}}{\gamma_{t}^{c}}=\frac{T-1}{2} \approx 0.419643
$$

where $\left|p_{1}\right|=\left|p_{2}\right|$ is defined in (4.1.18) (4.1.19) (4.1.20) which relates to the real valued part of the second eigenvalue of the tribonacci polynomial companion matrix, $M$, (4.1.5).

Decompositions of the snub cubical graph adjacency matrix (4.2.6) and decompositions of the corresponding 24-vertex spherical code, ${ }_{{ }^{*}}^{\star} C^{24},(4.1)$ are conjectured above to yield rich insights into the quantum gravity correspondence (4.2.5), and models of particle physics and cosmology. The cosmographic distance ratio (7.2) is proposed to be one of these and future research aims to discover more. 


\section{Astronomical Observations}

Astronomical observations are used to further discuss and test Tribonacci-QC. We have already discussed the good fits between the model and observations as they pertain to the present value of the Hubble parameter, $h_{t \rightarrow 1}$, (5.14) (5.16), the deceleration-acceleration transition redshift, $z_{d a},(5.21)$, the physical baryon density parameter, $\Omega_{b} h^{2}$, (6.1), baryon density parameter, $\Omega_{b}$, (6.2), matter density parameter, $\Omega_{m}$, (6.3), and cosmographic distance ratio, $r$, (7.2). These parameter-based fits are covered in above Sections 5, 6 and 7.

In this Section 8 we discuss astronomical observations framed by the above parameters, which also relate to and support the Tribonacci-QC model. Cosmological ages are specified in billions of years (Gyr) ago, as lookback times.

\subsection{Fast Radio Bursts}

The possibility of astronomical observations relating to phenomena originating before 13.8 Gyr is precluded in Big Bang singularity cosmology. However, in Tribonacci-QC there is no singularity at the time of last zero cosmological scale, $t_{a=0(-1)}$. In Tribonacci-QC this event, 13.8 Gyr ago, marks a zero crossing between negative scale and positive scale, where scale is relative to the optimal non-antipodal codes' spherical horizon. Negative scale projects inside the horizon, whereas positive scale projects outside the horizon (Section 5) (Figure 19).

Also recall that negative model time, $t<0$, marks an epoch of 24-spacetime. Any present astronomical observations pertaining to phenomena caused before 13.8 Gyr ago (model time, $t_{a=0} \approx-0.33174$ ) (5.6), indeed before $10.4 \mathrm{Gyr}$ ago (model time $t=0, z=2$ ), were configured with 24 degrees of freedom and then metamorphosed via a coarsening parallelization to our present 4(6) degrees of freedom in spacetime. We therefore seek quantum astrophysical structures that required 24 degrees of freedom during their synthesis, but which have since been coarsened within 4(6) degrees of freedom, in 4(6)-spacetime. We seek 24-dimensional astrophysics confined in a subsequent cage of 4(6)-spacetime.

Let us consider radioactive decay, related to random quantum vacuum fluctuations that promote relaxation to a lower energy state via quantum tunnelling. I propose Fast Radio Bursts (FRBs) originate in high energy 24-dimensional Planck stars which cannot be completely confined in cages (event horizons) of coarser 4(6)-spacetime, due to their excessive energy fluctuations. A graph model of such a cage is illustrated in Figure 13.

FRBs, in Tribonacci-QC, are violent quantum tunnelling events, cascading into strong millisecond radio bursts, caused during the decay of 24-dimensional Planck stars, which are incompletely confined by their 4(6)-dimensional event horizons. This radioactive decay process will ultimately fully confine these 24-dimensional astrophysical objects within their 4(6)-dimensional cages.

For a discussion related to this Tribonacci-QC interpretation, the reader is referred to seminal research by Rovelli, Vidotto and Barrau [44] [45]. 


\subsection{Black Holes and Deep Learning Cosmological Cycles}

Following the arrow of cosmological time, from the deep past towards the present, let us consider astronomical observations pertaining to the period 13.8 Gyr to 10.4 Gyr ago $(t=0, z=2)$. Recall Tribonacci- $Q C$ proposes this period occurs within dominantly 24-spacetime. In Big Bang cosmology this period is conventionally considered to comprise a 4-dimensional spacetime framework which covers the interval from the Planck epoch, through the quark epoch, hadron epoch, neutrino decoupling, lepton epoch, nucleosynthesis, photon epoch, recombination, the dark ages, then reionization to galaxy formation and the onset of galaxy clustering.

This Tribonacci-QC model seeks to reconcile with Big Bang cosmology as much as is reasonably practicable, given Big Bang cosmology's scientific maturity and the considerable weight of supporting evidence. However, tensions are known in Big Bang cosmology and so it is not yet a theory of everything that has earned total consensus.

A claim in Tribonacci-QC states that during this period $13.8 \mathrm{Gyr}$ to $10.4 \mathrm{Gyr}$ ago $(z=2, a(t)=1 / 3)$ astrophysical structures were generated in 24-spacetime. To observe these structures, post $a(t)=1 / 3$, we need to look through Einsteinian 4(6)-spacetime, into distant 24-spacetime.

Low mass primordial black holes [46] [55] [56] are hypothesised to originate from this period, beyond $z=2$, and may seed super massive black holes at the centres of galaxies in present 4(6)-spacetime. See also Kashlinsky's [50] analysis of what may have occurred if some primordial matter ("dark" matter in his analysis) comprised a population of primordial black holes. Such primordial black holes would distort the distribution of mass in the early universe, introducing a fluctuation with consequent effects when the first stars begin to form hundreds of millions of years later. Very old supermassive black holes have been observed and are in tension with standard Big Bang cosmology because their time of formation defies conventional explanation.

We may think of a black hole in terms of a further coarsening of the spherical code which is isomorphic to the dimensions of spacetime. Let us consider the formation of a black hole, wherein 4(6)-spacetime (tetrahedral spherical code in Figure 6) coarsens in two stages, as depicted in Figure 21. Firstly, the tetrahedral spherical code (Figure 6) coarsens to a 3-vertex spherical code (great circle equilateral triangle, with edges coloured light blue, see left side of Figure 21). One way it could do this would be for the time-like (yellow) vertices to advance to their subsequent adjacent vertex in the example Hamiltonian super-cycle (2.5). Thus, time-like (yellow) vertices 1 and 5 advance to the $x$ space-like dimension (red), vertices 6 and 7 advance to the $y$ space-like dimension (green) and vertices 8 and 11 advance to the $z$ space-like dimension (blue). A similar chromatic coarsening arrangement can be derived when the time-like (yellow) vertices shift to their prior adjacent vertex in the example Hamiltonian super-cycle (2.5). The 3 -vertex code is isomorphic to the dimensions of 3(8)-spacetime. I conjecture this 3-vertex code governs the physics of the event horizon of the black hole. 

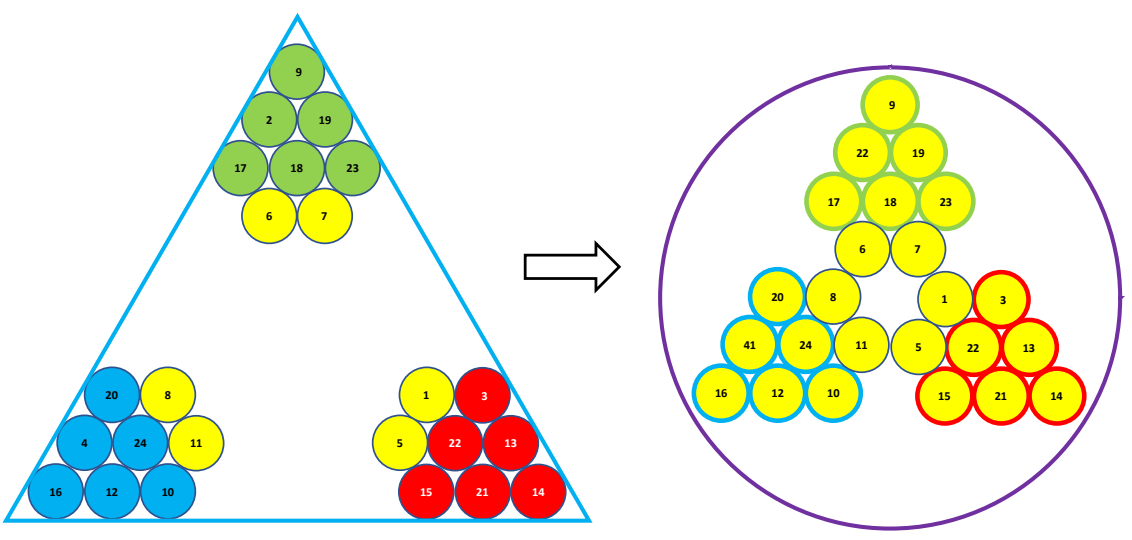

Figure 21. The example Hamiltonian super-cycle (2.5) is shown here on the left in a triangular perimeter (light blue edges), substantially as configured in Figure 6, with the main difference being that here the time-like (yellow) vertices are shifted from the apex of the former spherical tetrahedral graph to the triangular space-like base of this graph. This represents a graph coarsening (and corresponding spherical code coarsening) from the 4(6)-spacetime configuration in Figure 6 to a 3(8)-spacetime configuration in the left side of this figure. The left side of this figure may be thought of as the event horizon of a black hole. The right side of this figure shows the configuration after a further subsequent coarsening stage, through which the 3-vertex graph (left side) coarsens to a 1-vertex graph (right side), where the 24 vertices are parallelized into one ultrabroadband single degree of freedom, represented by all vertices parallelized within the enclosing purple circle. This purple circle should be thought of as one point, comprising 24 parallelized vertices. The right side of this figure represents 1(24)-spacetime, or eternal time within a black hole.

A second stage of coarsening of the spherical code is shown in the right side of Figure 21. In this second stage, the 3 -vertex spherical code (great circle equilateral triangle, with edges coloured light blue) coarsens to a 1-vertex spherical code (a point represented by a purple circle). The 1-vertex code is isomorphic to the dimensions of ultrabroadband 1(24)-spacetime. I conjecture this 1-vertex code governs the physics inside the event horizon of the black hole and that it has qualities which are those of eternal time (all vertices are yellow coloured, with their legacy space-like coloration depicted as red, green and blue coloured circles). The entire right side of Figure 21 is macroscopically manifest as eternally stretched time; however microscopically, it comprises 24 parallelized degrees of freedom.

In Section 8.3.7 below, on Wheelerian observers shaping our participatory universe, I propose that ultimate coarsening of apparent reality to ${ }_{\leftrightarrow}^{\star} C^{1}$, isomorphic to eternal time, is imminent for each observing agent in a Wheelerian participatory universe, as model time $t \rightarrow 1$. Referring to the right side of Figure 21 we may see in this diagram the eternal time configuration of the mortal observer whose quantum participatory life has finally concluded, upon taking its last individual experience, time attains its individual value, $t=1$.

Such 1-vertex sets (right side of Figure 21), representing black hole interiors and extinct quantum participatory life may be recycled as an information-rich feedstock to the left side of the Tribonacci-QC process depicted in Figure 2, 
which shows spherical code evolution as a computationally intelligent, deep learning, self-organization process. In this way, cosmological deep learning, fine-tuning and black hole to white hole rebirth [112] have parallel bases in the shared dimensions of Tribonacci-QC.

An alternative, or complementary, way to consider a black hole in Tribonac$c i-Q C$ is depicted in Figure 13. That figure represents the corresponding quantum mechanical configuration of a black hole, wherein the central cluster of 12 apical vertices are isomorphic to 12(2) degrees of freedom in $S U(5)$ inside the event horizon and the circumscribing 12 basal vertices (Figure 13) are isomorphic to the degrees of freedom in $S U(5)$ outside the event horizon. In concluding this sub-section, Tribonacci-QC provides novel graph-based models for astronomical observations associated with black holes, whether they formed in primordial cosmological times or more recently.

\subsection{Transition Event $(z=2)$}

It is important to consider that our present observations are being made in 4(6)-spacetime. When we observe beyond $z=2$, to study objects formed before 10.4 Gyr ago, we are looking through the medium of 4(6)-spacetime. Like an archerfish (Toxotidae), targeting airborne insects from underwater, our images of objects formed in distant precursor 24-spacetime, beyond $z=2$ and formed before 10.4 Gyr ago, are modified by refraction through our 4(6)-spacetime. Recall, Tribonacci-QC postulates that time advances 6-fold faster in 24-spacetime than in coarser 4(6)-spacetime. Snell's well-known law states that the ratio of the sines of the angles of incidence and refraction is equivalent to the ratio of phase velocities in juxtaposed media.

In Tribonacci-QC, we expect a refractive interface horizon at $z=2$, however as we generally observe perpendicular to this interface horizon, angular distortions are not extreme. Nonetheless, perturbations of the $z=2$ interface horizon, caused by quantum fluctuations during the transition from 24-spacetime to 4(6)-spacetime, are postulated to form refractive lenses. Recall also, the 6-fold difference in speeds of information exchange between juxtaposed states of spacetime across the $z=2$ interface horizon in the Tribonacci- $Q C$ model.

In addition to considering refraction, we need to consider the information-opacity of coarse 4(6)-spacetime to certain phenomena which share dimensions in deep $(z>2$ ) 24-spacetime and 12(2)-dimensional quantum mechanics. Observers presently in coarse 4(6)-spacetime, where their $t \rightarrow 1$, cannot resolve the finer micro-physics of these higher dimensional realms. Our coarse 4(6)spacetime is too grainy; it has a strong information-opacity which causes phenomena to appear classical. Consequently, distant astrophysical structures, where $z>2$, cannot be resolved in their dimensionally finer grained magnificence and the complex quantum mechanical realm appears fuzzy. To observe Nature's phenomena which share dimensions in deep $(z>2)$ 24-spacetime and 12(2)-dimensional quantum mechanics, measurements made in 4(6)-spacetime, 
where $t \rightarrow 1$, must strongly sharpen their resolution in both space and time. Indeed, it is via such a high resolution strategy that science is currently unlocking the marvels of quantum physics and the cosmology of deep spacetime.

Another important general characteristic of the $z=2$ event is that it occurs at $t=0$ in model time, (10.4 Gyr ago), that is when the first derivative of the cosmological scale factor $a(t)$ is infinite for an infinitesimal moment of transition time. From (5.1) the first derivative of the scale factor is given by:

$$
\begin{gathered}
\dot{a}(t)=3^{t^{T / 3}-2} t^{T / 3-1} T \log (3) \\
\lim _{t \rightarrow 0^{+}} \dot{a}(t)=\infty
\end{gathered}
$$

In the discrete Tribonacci- $Q C$ model this quantum transformation (8.3.2) event is a high energy shock-spike, or bow shock, concurrent with the transition from 24-spacetime to 4(6)-spacetime. In the following sub-sections, we consider phenomena caused by this $z=2$ high energy shock-spike, or bow shock.

\subsubsection{Peak Star formation Rate Density $(z=2)$}

The Fermi Large Area Telescope Collaboration 2018 [31] reports that the light emitted by all galaxies over the history of the Universe produces the extragalactic background light, at infrared, optical and ultraviolet wavelengths. This extragalactic background light is a source of opacity for $\gamma$-rays via photon-photon interactions, leaving a record in the spectra of distant $\gamma$-ray sources. They measured this attenuation using over seven hundred active galaxies and one $\gamma$-ray burst. This analysis allows them to explain the evolution of the extragalactic background light and determine the star-formation history of the Universe, over $90 \%$ of cosmic time. Their star formation history is consistent with independent measurements from galaxy surveys, which clearly peaks at redshift $z=2$. See also [52].

In the context of Tribonacci-QC peak star formation rate density relates to the coarsening transition from 24-spacetime to 4(6)-spacetime at $z=2$. Consider triggered star formation during which events compress a molecular cloud initiating gravitational collapse [53]. Molecular clouds may collide or a supernova explosion may trigger cloud collapse, by sending shocked matter into it at high velocity. The new stars thus born may then produce supernovae, leading to self-propagating star formation.

A source of gravitational shock throughout the entire cosmos is proposed to be the abrupt (8.3.1) (8.3.2) coarsening transition from 24-spacetime to 4(6)-spacetime at $z=2$. Referring to Figure 18, we see the cosmological scale factor at $t=0$, $a(t)=1 / 3, z=2$ passes through an abrupt positive inflection with an instantaneously infinite rate of expansion $\dot{a}(t) \rightarrow \infty$ over an infinitesimal time interval $\delta t$ (8.3.1) (8.3.2). This is proposed to be a direct signature in Tribonac$c i-Q C$ of a $z=2$ transition gravitational shock having an abrupt transition effect on the scale of the entire cosmos. The model informs us that gravitational binding of the cosmos spontaneously weakens upon coarsening from 24-spacetime to 4(6)-spacetime which is manifest in an instantaneous inflation of cosmologi- 
cal scale at $t=0$. From general relativity, time dilates with weakening gravity. This aligns with the principle in Tribonacci-QC that time advances 6-fold more slowly in 4(6)-spacetime than in the preceding 24-spacetime.

Consider the transition from 24-spacetime to coarser 4(6)-spacetime as a cosmological flash expansion process. It is a flash expansion which accompanies a gravitational high energy shock-spike, or bow shock, throughout the cosmos and causes the star formation rate density to peak at $z=2$ via standard model molecular cloud compression mechanisms.

\subsubsection{Peak Quasar Activity $(z=2)$}

A quasar, or quasi-stellar object (QSO), is an extremely luminous active galactic nucleus. Peak QSO and peak star formation activity are concomitant. Croom et al. [32] highlight that the conditions in active galactic nuclei at $z=2$ are fundamentally distinct from those at lower redshift, necessitating at least an order-of-magnitude change in the Eddington luminosity ratio, or in the mass of the black hole relative to the galaxy's bulge mass. They conclude that either the fuelling efficiency is much greater at high redshift, or that more massive black holes are active at high redshift. Both conclusions point to stronger gravitational interactions before $z=2$, which fits with stronger gravity in the 24-spacetime state of Tribonacci-QC. Peak QSO activity at $z=2$ is claimed to be caused by the $z=2$ high energy shock-spike, or bow shock, (8.3.1) (8.3.2) throughout the cosmos. See also [33].

An important recent paper by Risaliti and Lusso [54] presents measurements of the expansion rate of the Universe based on a Hubble diagram of large number of quasars. In their paper, an informative plot of the distance modulus (ordinate) versus redshift, $z$, (abscissa) depicts a reasonably organised scatter of data points. The scatter of points on their distance modulus versus redshift plot in the low redshift range, $0<z<2$, has a distinctive pattern in agreement with the concordance $\Lambda \mathrm{CDM}$ model of cosmology.

However, the scatter of points on the same distance modulus versus redshift plot in the higher redshift range, $2<z<5.5$, has a less organised pattern which nevertheless credibly deviates from the concordance $\Lambda$ CDM model of cosmology. Risaliti and Lusso determine consistency with supernovae data and the concordance $\Lambda \mathrm{CDM}$ model of cosmology up to $z=1.4$. The author suggests our readers could also reasonably interpret, from the raw data points of Risaliti and Lusso [54], a Tribonacci-QC transition evident at $z=2$ in the scatter of points on their distance modulus versus redshift plot.

\subsubsection{Environmental Quenching $(z=2)$}

Ji et al. [49] discuss evidence of environmental quenching at redshift $z \approx 2$.

At redshift $z=2$, they conclude quiescent galaxies cluster around other quiescent galaxies much more strongly than the general galaxy population of the same stellar mass, at the same redshift and about two times more than galaxies hosting more massive halos. 
It is proposed, in Tribonacci-QC, that such environmental quenching is an emergent phenomenon, caused by the $z=2$ high energy shock-spike, or bow shock, (8.3.1) (8.3.2) throughout the cosmos, concomitant with the 24-spacetime to $4(6)$-spacetime transition.

\subsubsection{CMB Lensing $(z=2)$}

Recall, perturbations of the $z=2$ interface horizon, caused by quantum fluctuations during the transition from 24-spacetime to 4(6)-spacetime, are postulated to form refractive lenses. Consider, for a flat universe, the cosmic microwave background (CMB) lensing kernel also peaks at $z=2$ [34] which raises the possibility of an equivalence, at this redshift, between CMB lensing and Tribonacci-QC refraction lensing.

\subsubsection{GRB Large Scale Structure $(z=2)$}

A distinctive Gamma-Ray Burst (GRB) cluster at $z \approx 2$ covers almost one-eighth of the sky, spanning half of the constellations of Bootes, Draco, and Lyra, and all the constellations of Hercules and Corona Borealis. This structure is called the Hercules-Corona Borealis Great Wall. Its discoverers, Horváth et al. [47] [48] estimate it to be about 2000 to $3000 \mathrm{Mpc}$ across. This structure is 20 to 30 times larger than the $100 \mathrm{Mpc}$ scale of the so-called "End-of-Greatness" [51] and thus defies explanation in standard cosmology. The End-of-Greatness is the scale, in standard cosmology, where the unevenness seen in the structure of the universe is isotropized and homogenized in accord with the Cosmological Principle.

In this Tribonacci-QC model there is no such expectation for any End-ofGreatness at $100 \mathrm{Mpc}$. Indeed, to the contrary, there is an expectation that the cosmological scale transition, at the $z=2$ dimensional coarsening event, would have shocked the cosmos and left bright tell-tale astronomical anomalies, clustered on a very large scale, at that redshift. GRBs are the most luminous form of normal matter energy release, which fits with the gargantuan energy shock-spike, or bow shock, caused by the $z=2$ dimensional coarsening (8.3.1) (8.3.2).

\subsubsection{Peak ${ }^{6} \mathrm{Li}$ Genesis by Cosmic Rays $(z=2)$}

Observed ${ }^{6} \mathrm{Li}$ is over abundant by a factor of 1000 , which poses a serious challenge for the standard Big Bang nucleosynthesis model [35]. Nath et al. discuss a model in which cosmic rays simultaneously produce the observed over-abundance of ${ }^{6} \mathrm{Li}$ and pre-heat ambient gas to explain X-ray observations of galaxy clusters. They attempt to explain the over-abundance of ${ }^{6} \mathrm{Li}$ at $z=2$ which corresponds to the formation epoch of halo metal-poor stars and to an increased entropy level $\left(\sim 300 \mathrm{keV} \cdot \mathrm{cm}^{2}\right)$ needed to explain X-ray observations of galaxy clusters. They relate the two sets of observations; enhanced ${ }^{6} \mathrm{Li}$ abundance in metal-poor stars and enhanced entropy in gas in galaxy clusters, by way of cosmic rays from active galactic nuclei [36].

As proposed by Suzuki and Inoue [37] cosmic ray production of ${ }^{6} \mathrm{Li}$ by struc- 
ture formation shocks as a dissipative process during galaxy formation offers a different model. They associate their process to the peak star formation rate and to the merger of sub-galactic clumps into galaxies (whereby the majority of in-fall kinetic energy is virialized) at $z=2$. Such gravitational shocks induced by infalling and merging sub-galactic clumps (during hierarchical structure formation of a galaxy) could dissipate enough energy, so they propose, and cosmic rays accelerated by such shocks provide a natural explanation of the observed over-abundance of ${ }^{6} \mathrm{Li}$. See also [38].

Whether or not these models are completely successful, key points pertinent to Tribonacci-QC are that there is an over-abundance of ${ }^{6} \mathrm{Li}$ somehow related to redshift $z=2$, whilst corresponding causal cosmic rays from active galactic nuclei or gravitational shocks or something else are required to augment standard Big Bang nucleosynthesis models. Tribonacci- $Q C$ provides an explanation for a requisite high energy shock-spike, or bow shock, (8.3.1) (8.3.2) concomitant with the 24-spacetime to 4(6)-spacetime transition event.

Consider that ${ }^{6} \mathrm{Li}$ is fundamental to conscious quantum information processing in the minds of sentient agents [3]. In the following Sub-Section 8.3.7 we discuss such sentient agents as Wheelerian participatory universe observers [5]. In other words, through Darwin's universal algorithm, our quantum deep learning cosmos [1] Naturally selects the advantageous constituents required to build sentience; to participate, to observe itself and to cause effects on its matter distribution [2].

I propose the observed ${ }^{6} \mathrm{Li}$ over-abundance is generated by cosmic rays via parallel astrophysical processes, including those proposed by [35] [36] [37], which all peak at the transition event $z=2$.

More significantly, it is the Tribonacci- $Q C$ transition event at $z=2$, when 24-spacetime coarsens 6-fold into 4(6)-spacetime, which is closer to the root cause of the emergent parallel astrophysical processes. In this concept, the transition event at $z=2$ is a trigger, but what, or more aptly who, pulled that trigger? In a deep learning cosmology, founded on epistemic drive, such as presented in this paper, a chief suspect is whoever acquires advantageous knowledge via the Natural resources of ${ }^{6} \mathrm{Li}$. Cosmological information processing feedback loops (recall Sub-Section 8.2) in Tribonacci-QC existentially include conscious observers, who select outcomes and build consensus via communication, and they are chief suspects for pulling the $z=2$ transition trigger (Sub-Section 8.3.7).

The singularity problem at $t=0$ is removed by a cosmological quantum deep learning self-measurement event that is a universal decoherence, transferring information from the precursor real and imaginary quantum state $(z>2)$ universe with 24-dimensions (left side of Figure 18), to the real and coarsened 4(6)-spacetime environment, with microscopically circumscribed 12(2)-dimensional quantum physics (right side of Figure 18).

The "Big Bang" singularity problem is solved with a "Get Real" cosmological quantum self-measurement event by the quantum deep learning cosmos. This 
event is epistemically driven in a runaway self-realisation, with positive feedback through cosmological cycles, propelled by physics but directionally nudged by its own emergent agents. In other words, the Agency of Life triggers Schrödinger's cat to pounce out of its box and fine-tunes the fundamental parameters of $\mathrm{Na}$ ture through evolutionary cosmological cycles.

\subsubsection{Wheelerian Observers Shape Our Participatory Universe $(z=2)$} Wheeler explains his seminal idea of observer-participancy by describing the world as a self-synthesizing, quantum networking, system of existences [5] and writes about it in terms of the coming explosion of life. I subscribe to the idea of Wheelerian observers shaping our participatory universe, though I wind the clock back to $10.4 \mathrm{Gyr}$ ago ( $z=2$ ), that is, to First Life in the universe (long before first Life on Earth).

By First Life I mean the emergence of outcome-selecting biological life in the cosmos. Stephen Hawking argued that the necessary planetary conditions for our form of intelligent life first occurred some $10 \mathrm{Gyr}$ ago [39] and this estimate fits well with my Tribonacci-QC model and with Wheelerian observers shaping our participatory universe since First Life. Indeed, I claim First Life is fundamentally and causally connected to the $z=2$ transition event. Peak ${ }^{6} \mathrm{Li}$ genesis, First Life, and the 24-spacetime to 4(6)-spacetime transition are coeval, parallel-optimized, outcomes of multiple deep-learning cosmological cycles (section 8.2) which preceded our current cycle.

Life, even microbial, is outcome-selecting. The onset of outcome-selection marks the dawn of observer-participancy and Agency and the beginning of its cascading influence over the quantum information structure of our entangled Universe.

We know from fossil evidence that Life initiated on Earth about 3.5 Gyr ago, only some $0.5 \mathrm{Gyr}$ after the Earth became stable and cool enough for life to develop and evolve. There has therefore been plenty of time since the $z=2$ transition event for three back-to-back episodes of similar evolution towards sentience elsewhere in the cosmos. Now consider the potential for parallel biogenesis and biological evolution across innumerable life-bearing planets and moons and we reach the conclusion that observer-participancy, including observer-observer communication (e.g. observer-consensus about constellations and the workings of physics), has the capacity to physically shape the cosmos. Add to that my proposal that the cosmos is deep-learning and is in a present, parallel-opitmized, cycle which was preceded by innumerable prior cycles. Furthermore, that optimization is considered to occur across the shared dimensions of Tribonacci-QC.

Indeed, in the language of Schrödinger, and in Tribonacci-QC, I envisage First Life collapsing the wave function of 24-spacetime, from its superposed eigenstates, through widespread participatory observation into a 4(6)-spacetime eigenstate. I consider the pervasiveness of participating, communicating and consensus-building observers as being an omnipresent Agency. This Agency is in- 
trinsic to the environment for decoherence and thus einselection occurs via its ever-present outcome-selection and broadband, consensus-affirming, classical outcomes.

Finally, consider that Life occupies and fulfils a pivotal participatory position amidst the scale of everything, which spans from the miniscule quantum mechanical dominion, to the vastness of our relativistic cosmos. I claim Life provides existential outcome-selecting Agency, spanning all scales, and operates at the measurement-nexus of a preposterous scale hierarchy. See Paul Davies' recent book, "The Demon in the Machine: How Hidden Webs of Information Are Finally Solving the Mystery of Life" [113], for an inciteful overview of the connections between physics and biology in terms of information science, and for a useful bibliography.

So, surely the configuration of Life itself also obeys the information rules of Tribonacci-QC? Indeed, I claim it does and most pertinently at the instant of abiogenesis, at $z=2$, when physical chemistry first flips into Life. DNA is proposed in Tribonacci-QC to have evolved from an $8 \times 3$-nucleotide (eight codon) primeval molecular code on the model's 24 shared dimensions.

Reconsider Figure 2, middle left, where eight 3-vertex codes (equilateral triangle on a great circle) are illustrated to self-organise into one 24-vertex code (chiral, spherical snub cube), middle right. Also reconsider Figure 4, which shows eight snub cubical graphs in triangular perimeters with eight 3-vertex sets (solid black dots) that self-organize into the 24-vertex set. Now reconsider a Hamiltonian super-cycle in that 24-vertex set, e.g. (2.5).

I propose the eight 3-vertex sets are isomorphic to eight codons (sequences of 3 nucleotides in a unit of genetic code). They self-organise to constitute a snub cubical 24-vertex set, with a Hamiltonian super-cycle which I claim is isomorphic to the most primeval molecular algorithmic-loop, which is Nature's prebiotic precursor to DNA (and RNA).

Thus Tribonacci-QC proposes that quantum mechanics, abiogenesis and cosmology are all governed by the 24 shared dimensions of snub cubical, optimal, non-antipodal spherical codes, operating at three inter-related entangled scales across a preposterous hierarchy, with Life being the pivotal measurement-nexus, i.e. the participatory observer (Schrödinger's box opener), in the quantum information processing of our entire Universe.

\section{Summary}

In this paper it is proposed that the discrete dimensions of our quantum information world are the most fundamental foundations of everything. A dimension is a structure that categorizes discrete information. Minimising computational time, a shared dimension makes economic computational use of that degree of freedom, as a natural resource for the broadband parallel processing of distinguishable information. I focus on the Natural selection, evolution and function of shared dimensions in a deep learning quantum cosmology model. 
These shared dimensions are conformed dimensions in computer science, which ensure consistency between Nature's quantum information processing of its physics at macro, meso and microscopic scales, and ensure consistency spanning from deep evolutionary time, through observer lifetimes, to near-instantaneous causal particle physics. Nature's quantum computations at the cosmological scale can share dimensions with its computations at the Planck scale because the extreme difference in scale (a preposterous hierarchy) protects against detrimental crosstalk. Nature's two extreme scales come into Reality at the meso-scale nexus of the sentient Wheelerian participatory observer.

The Tribonacci-QC model presented proposes our deep learning quantum computational universe is founded on a common architecture of shared dimensions, defined by discrete and exceptional spherical codes, on a 2-sphere, $S^{2}$. The shared dimensions are utilised by Nature to compute the entirety of physics, across the whole universe, at all scales. The information processed in these shared dimensions is parallelized, in coordinated superpositions, and decomposes into Nature's innumerable emergent physical phenomena, through observations described by fundamental and effective theories.

This Tribonacci-QC model invokes four optimal $\star$, non-antipodal $\leftrightarrow$, spherical codes, ${ }_{\leftrightarrow}^{\star} C \subset S^{2}: 1$-vertex, 3-vertex (great circle equilateral triangle), 4-vertex (spherical tetrahedron) and 24-vertex (spherical snub cube). The vertices are einselected centres of coherent quantum information that maximise their minimum separation and survive environmental decoherence on a noisy horizon. Twenty-four 1-vertex codes, $24 \times{ }_{\leftrightarrow}^{\star} C^{1}$, self-organize into eight 3-vertex codes, $8 \times{ }_{\leftrightarrow}^{\star} C^{3}$, which self-organize into one 24-vertex code, ${ }_{\leftrightarrow}^{\star} C^{24}$, isomorphic to dimensions of 24-spacetime and the 12(2), or 24, generators of $S U(5)$.

Snub cubical 24-vertex code chirality causes matter asymmetries and the corresponding graph-stress has normal and shear components relating to respective sides of Einstein's tensor equivalence $G_{\mu \nu}=\kappa \mathcal{T}_{\mu \nu}$. Cosmological scale factor and Hubble parameter evolution is formalized as an Ostwald-coarsening function of time, scaled by the tribonacci constant $(T \approx 1.839)$ property of the snub cube. The 24-vertex code coarsens to a broadband 4-vertex code, isomorphic to emergent 4-spacetime and antecedent structures in 24-spacetime metamorphose to familiar 4-spacetime forms. Each of the coarse code's 4-vertices has 6-fold parallelized degrees of freedom (conserved from the 24-vertex code), ${ }_{\leftrightarrow}^{\star} C^{4(6)}$, so 4-spacetime is properly denoted 4(6)-spacetime.

Cosmological parameters are formalized: $\mathrm{CMB}$

$h=H_{0} / 100=T \log (3) / 3 \approx 0.674$, Distance Ladder $h=4 T \log (3) / 11 \approx 0.735$, $z_{d a}=3 \mathrm{e}^{1-3 / T}-1 \approx 0.596, \Omega_{b} h^{2}=\arccos (\sqrt{T / 2}) / 4 \pi \approx 0.023$ and $\gamma_{t}^{o} / \gamma_{t}^{c}=(T-1) / 2 \approx 0.420$. Due to 6 -fold parallelization, the total matter density parameter is 6-fold heavier than the baryon density parameter, $\Omega_{m}=6 \Omega_{b}=27 \arccos (\sqrt{T / 2}) / 2 \pi \log ^{2}(3) T^{2} \approx 0.302$. A torrent of information-equivalent energy downloads from 6-fold faster 24-spacetime to 4(6)-spacetime. Consequent stress on 4(6)-spacetime causes it to resize its dynamic memory, 
expanding its cosmological scale. Ultimate coarsening of apparent reality to ${ }_{\leftrightarrow}^{\star} C^{1}$, isomorphic to eternal time, is imminent for each observing agent in a Wheelerian participatory universe. Life, even microbial, is outcome-selecting. The onset of outcome-selection marks the dawn of observer-participancy and Agency, and the beginning of its cascading influence over the quantum information structure of our entangled Universe. DNA is proposed to have evolved from an $8 \times 3$-nucleotide (eight codon) primeval molecular code on the model's 24 shared dimensions.

The significance of the model's cosmological transition event, from 24-spacetime to 4(6)-spacetime is emphasized. It occurs at redshift $z=2$, that is at model time $t=0$, when the scale factor $a(t)=1 / 3$. This aspect of the Tribonacci-QC model clearly distinguishes it from other models. I present an introductory case for the extraordinariness of redshift $z=2$ in astronomy. This redshift marks an event which is related to several phenomena; including peak star formation rate density, quasar activity, $\mathrm{CMB}$ lensing, ${ }^{6} \mathrm{Li}$ genesis by cosmic rays and exoplanetary abiogenesis of Wheelerian participatory universe observers.

This model also makes certain testable assertions about the dimensionality of particle physics. Tribonacci-QC describes a quantum mechanical realm for particle physics which has 12(2) degrees of freedom and claims these dimensions, which are shared with those of 24-spacetime, are circumscribed within the coarsened 4(6)-spacetime. Furthermore 12 of these are Naturally selected whilst 12 are de-selected yet virtual. Exceptional, self-organizing spherical codes, that are simultaneously optimal and non-antipodal, are isomorphic to the shared dimensions which provide degrees of freedom in Nature's entangled quantum information processing of General Relativity, Darwinian Life and Quantum Mechanics. This feature of Tribonacci-QC presents a novel path towards their long-sought unification.

\section{Conclusions}

Having set out a summary in the above Section 9, I now succinctly conclude with some overarching high-level reflections about this Tribonacci- $Q C$ model. These can be viewed from three perspectives.

Firstly, the most significant structure of our quantum computational Universe is its fundamental dimensionality, which in this model is a dimensionality shared across all scales. The preposterous hierarchy between quantum mechanics and relativistic cosmology is bridged by the meso-scaled Agency of Life, the participatory observers who collapse wave functions and bring about Reality, all of which can be related to the same shared dimensions. Indeed, it is the preposterous hierarchy of scale which allows for sharing of dimensions without detrimental crosstalk between its end members.

Secondly, the factor pairs of 24 have innate utility in the description of Nature. This is particularly where they characterise optimal, non-antipodal, spherical codes (self-isolated against detrimental crosstalk) and corresponding graphs. 
The factor pairs of 24 characterise quantum particle physics, a prebiotic precursor of DNA, and the geometrogenesis of spacetime (all on 24 shared dimensions, with some factors parallelized and Naturally selected in certain situations).

Thirdly, the 24-vertex spherical code which is optimal and non-antipodal, is snub cubical, and this configuration is intrinsically related to the tribonacci constant and its recurrence sequence. The tribonacci sequence innately regulates and rectifies chaos into orderly expansion which underpins such fundamental characteristics of our emergent Universe. Remarkably, many cosmological parameters can be simply expressed in terms of the tribonacci constant.

Shared dimensions, factor pairs of 24 and the tribonacci constant are Naturally mutual to this original model of our World.

\section{Acknowledgements}

This original research is self-funded and I thank my reviewers and editors for their valuable support.

\section{Conflicts of Interest}

The author declares no conflicts of interest regarding the publication of this paper.

\section{References}

[1] McCoss, A. (2016) Quantum Deep Learning Triuniverse. Journal of Quantum Information Science, 6, 223-248. https://doi.org/10.4236/jqis.2016.64015

[2] McCoss, A. (2017) Agency of Life, Entropic Gravity and Phenomena Attributed to "Dark Matter". Journal of Quantum Information Science, 7, 67-75. https://doi.org/10.4236/jqis.2017.72007

[3] McCoss, A. (2017) Lithium Quantum Consciousness. Journal of Quantum Information Science, 7, 125-139. https://doi.org/10.4236/jqis.2017.74010

[4] McCoss, A. (2018) It from Qutrit: Braided Loop Metaheuristic. Journal of Quantum Information Science, 8, 78-105. https://doi.org/10.4236/jqis.2018.82006

[5] Wheeler J.A. (1988) World as System Self-Synthesized by Quantum Networking. In: Agazzi, E., Ed., Probability in the Sciences, Synthese Library (Studies in Epistemology, Logic, Methodology, and Philosophy of Science), Vol. 201, Springer, Dordrecht, 103-129. https://doi.org/10.1007/978-94-009-3061-2_7

[6] Conway, J.H. and Sloane, N.J.A. (2013) Sphere Packings, Lattices and Groups, Vol. 290. Springer Science \& Business Media, New York.

[7] Tersoff, J., Teichert, C. and Lagally, M.G. (1996) Self-Organization in Growth of Quantum Dot Superlattices. Physical Review Letters, 76, 1675. https://doi.org/10.1103/PhysRevLett.76.1675

[8] Murray, C.B., Kagan, C.R. and Bawendi, M.G. (1995) Self-Organization of CdSe Nanocrystallites into Three-Dimensional Quantum Dot Superlattices. Science, 270, 1335-1338. https://doi.org/10.1126/science.270.5240.1335

[9] Konopka, T., Markopoulou, F. and Smolin, L. (2006) Quantum Graphity. https://arxiv.org/abs/hep-th/0611197

[10] Markopoulou, F. (2009) Space Does Not Exist, So Time Can. 
https://arxiv.org/abs/0909.1861

[11] Markopoulou, F. (2012) The Computing Spacetime. In: Cooper, S.B., Dawar, A. and Löwe, B., Eds., How the World Computes. CiE 2012. Lecture Notes in Computer Science, Vol. 7318, Springer, Berlin, Heidelberg, 472-484.

https://doi.org/10.1007/978-3-642-30870-3_48

[12] Żenczykowski, P. (2018) Quarks, Hadrons, and Emergent Spacetime. Foundations of Science, 1-19. https://doi.org/10.1007/s10699-018-9562-2

[13] Mikhalkin, G. (2006) Tropical Geometry and Its Applications. https://arxiv.org/abs/math/0601041

[14] Maclagan, D. and Sturmfels, B. (2015) Introduction to Tropical Geometry, Vol. 161. American Mathematical Society, Providence. https://doi.org/10.1090/gsm/161

[15] Finster, F. and Schiefeneder, D. (2013) On the Support of Minimizers of Causal Variational Principles. Archive for Rational Mechanics and Analysis, 210, 321-364. https://doi.org/10.1007/s00205-013-0649-1

[16] Schiefeneder, D. (2011) On Minimizers of Causal Variational Principles. Ph.D. Thesis, Universität Regensburg, Regensburg. https://epub.uni-regensburg.de/21629/1/dissertation.pdf

[17] Finster, F., Grotz, A. and Schiefeneder, D. (2012) Causal Fermion Systems: A Quantum Space-Time Emerging from an Action Principle. In: Finster, F., Müller, O., Nardmann, M., Tolksdorf, J. and Zeidler, E., Eds., Quantum Field Theory and Gravity, Springer, Basel, 157-182. https://doi.org/10.1007/978-3-0348-0043-3_9

[18] Baez, J. and Huerta, J. (2010) The Algebra of Grand Unified Theories. Bulletin of the American Mathematical Society, 47, 483-552. https://doi.org/10.1090/S0273-0979-10-01294-2

[19] Baez, J. (2008) My Favourite Numbers: 24. The Rankine Lectures. Glasgow Mathematical Journal Trust. https://www.youtube.com/watch?v=vzjbRhYjELo

[20] Weisstein, E.W., Snub Cube. From MathWorld. A Wolfram Web Resource. http://mathworld.wolfram.com/SnubCube.html

[21] Martini, B.W.H. (2002) On the Chiral Archimedean Solids. Contributions to Algebra and Geometry, 43, 121-133. http://emis.ams.org/journals/BAG/vol.43/no.1/b43h1wem.pdf

[22] Florek, W. (2018) A Class of Generalized Tribonacci Sequences Applied to Counting Problems. Applied Mathematics and Computation, 338, 809-821. https://doi.org/10.1016/j.amc.2018.06.014

[23] Basu, M. and Das, M. (2014) Tribonacci Matrices and a New Coding Theory. Discrete Mathematics, Algorithms and Applications, 6, Article ID: 1450008. https://doi.org/10.1142/S1793830914500086

[24] Cerda-Morales, G. (2018) The Unifying Formula for All Tribonacci-Type Octonions Sequences and their Properties. https://arxiv.org/abs/1807.04140

[25] Cerda-Morales, G. (2017) On a Generalization for Tribonacci Quaternions. Mediterranean Journal of Mathematics, 14, 239. https://doi.org/10.1007/s00009-017-1042-3

[26] Günaydin, M. and Gürsey, F. (1973) Quark Structure and Octonions. Journal of Mathematical Physics, 14, 1651-1667. https://doi.org/10.1063/1.1666240

[27] Dixon, G.M. (2013) Division Algebras: Octonions Quaternions Complex Numbers and the Algebraic Design of Physics, Vol. 290. Springer Science \& Business Media, New York.

[28] Furey, C. (2016) Standard Model Physics from an Algebra? 
https://arxiv.org/abs/1611.09182

[29] Furey, C. (2015) Charge Quantization from a Number Operator. Physics Letters B, 742, 195-199. https://doi.org/10.1016/j.physletb.2015.01.023

[30] Furey, C. (2014) Generations: Three Prints, in Colour. Journal of High Energy Physics, 2014, 46. https://doi.org/10.1007/JHEP10(2014)046

[31] Fermi-LAT Collaboration (2018) A Gamma-Ray Determination of the Universe's Star Formation History. Science, 362, 1031-1034.

https://doi.org/10.1126/science.aat8123

[32] Croom, S.M., Schade, D., Boyle, B.J., Shanks, T., Miller, L. and Smith, R.J. (2004) Gemini Imaging of QSO Host Galaxies at $\mathrm{z} \sim 2$. The Astrophysical Journal, 606, 126-138.

[33] Glikman, E., Simmons, B., Mailly, M., Schawinski, K., Urry, C.M. and Lacy, M. (2015) Major Mergers Host the Most-Luminous Red Quasars at z 2: A Hubble Space Telescope WFC3/IR Study. The Astrophysical Journal, 806, 218.

[34] Hand, N., Leauthaud, A., Das, S., Sherwin, B.D., Addison, G., Bond, J.R., et al. (2015) First Measurement of the Cross-Correlation of CMB Lensing and Galaxy Lensing. Physical Review D, 91, Article ID: 062001. https://doi.org/10.1103/PhysRevD.91.062001

[35] Nath, B.B., Madau, P. and Silk, J. (2006) Cosmic Rays, Lithium Abundance and Excess Entropy in Galaxy Clusters. Monthly Notices of the Royal Astronomical Society: Letters, 366, L35-L39. https://doi.org/10.1111/j.1745-3933.2005.00127.x

[36] Abramowski, A., Aharonian, F., Benkhali, F.A., Akhperjanian, A.G., Angüner, E.O., Backes, M., et al. (2016) Acceleration of Petaelectronvolt Protons in the Galactic Centre. Nature, 531, 476-479. https://doi.org/10.1038/nature17147

[37] Suzuki, T.K. and Inoue, S. (2002) Cosmic-Ray Production of ${ }^{6} \mathrm{Li}$ by Structure Formation Shocks in the Early Milky Way: A Fossil Record of Dissipative Processes during Galaxy Formation. The Astrophysical Journal, 573, 168-173.

[38] Fields, B.D. and Prodanović, T. (2005) ${ }^{6} \mathrm{Li}$ and Gamma Rays: Complementary Constraints on Cosmic-Ray History. The Astrophysical Journal, 623, 877-888.

[39] Hawking, S. (1996) Life in the Universe. Public Lecture. http://www.hawking.org.uk/life-in-the-universe.html

[40] Forbes, D.A., Pastorello, N., Romanowsky, A.J., Usher, C., Brodie, J.P. and Strader, J. (2015) The SLUGGS Survey: Inferring the Formation Epochs of Metal-Poor and Metal-Rich Globular Clusters. Monthly Notices of the Royal Astronomical Society, 452, 1045-1051. https://doi.org/10.1093/mnras/stv1312

[41] Wang, F.Y. and Dai, Z.G. (2006) Constraining Dark Energy and Cosmological Transition Redshift with Type Ia Supernovae. Chinese Journal of Astronomy and Astrophysics, 6, 561-571. https://doi.org/10.1088/1009-9271/6/5/08

[42] Cunha, J.V. and Lima, J.A.S. (2008) Transition Redshift: New Kinematic Constraints from Supernovae. Monthly Notices of the Royal Astronomical Society, 390, 210-217. https://doi.org/10.1111/j.1365-2966.2008.13640.x

[43] Miyatake, H., Madhavacheril, M.S., Sehgal, N., Slosar, A., Spergel, D.N., Sherwin, B. and van Engelen, A. (2017) Measurement of A Cosmographic Distance Ratio with Galaxy and Cosmic Microwave Background Lensing. Physical Review Letters, 118, Article ID: 161301. https://doi.org/10.1103/PhysRevLett.118.161301

[44] Barrau, A., Rovelli, C. and Vidotto, F. (2014) Fast Radio Bursts and White Hole Signals. Physical Review D, 90, Article ID: 127503.

https://doi.org/10.1103/PhysRevD.90.127503 
[45] Rovelli, C. and Vidotto, F. (2014) Planck Stars. International Journal of Modern Physics D, 23, Article ID: 1442026. https://doi.org/10.1142/S0218271814420267

[46] Cai, Y.F., Tong, X., Wang, D.G. and Yan, S.F. (2018) Primordial Black Holes from Sound Speed Resonance during Inflation. Physical Review Letters, 121, Article ID: 081306. https://arxiv.org/abs/1805.03639 https://doi.org/10.1103/PhysRevLett.121.081306

[47] Horváth, I., Hakkila, J. and Bagoly, Z. (2014) Possible Structure in the GRB Sky Distribution at Redshift Two. Astronomy \& Astrophysics, 561, L12-L15. https://doi.org/10.1051/0004-6361/201323020

[48] Horváth, I., Bagoly, Z., Hakkila, J. and Tóth, L.V. (2015) New Data Support the Existence of the Hercules-Corona Borealis Great Wall. Astronomy \& Astrophysics, 584, A48-A55. https://doi.org/10.1051/0004-6361/201424829

[49] Ji, Z., Giavalisco, M., Williams, C.C., Faber, S.M., Ferguson, H.C., Guo, Y., et al. (2018) Evidence of Environmental Quenching at Redshift z 2. https://arxiv.org/abs/1806.04142

[50] Kashlinsky, A. (2016) LIGO Gravitational Wave Detection, Primordial Black Holes, and the Near-IR Cosmic Infrared Background Anisotropies. The Astrophysical Journal Letters, 823, L25. https://doi.org/10.3847/2041-8205/823/2/L25

[51] Kirshner, R.P. (2002) The Extravagant Universe: Exploding Stars, Dark Energy and the Accelerating Cosmos. Princeton University Press, Princeton, 71. https://press.princeton.edu/titles/7327.html

[52] Madau, P. and Dickinson, M. (2014) Cosmic Star-Formation History. Annual Review of Astronomy and Astrophysics, 52, 415-486. https://doi.org/10.1146/annurev-astro-081811-125615

[53] Prialnik, D. (2000) An Introduction to the Theory of Stellar Structure and Evolution. Cambridge University Press, Cambridge.

http://www.cambridge.org/9780521866040

[54] Risaliti, G. and Lusso, E. (2019) Cosmological Constraints from the Hubble Diagram of Quasars at High Redshifts. Nature Astronomy, 3, 272-277.

[55] Zel'dovich, Y.B. and Novikov, I.D. (1966) The Hypothesis of Cores Retarded during Expansion and the Hot Cosmological Model. Astronomicheskii Zhurnal, 43, 758. http://adsabs.harvard.edu/full/1967SvA....10..602Z

[56] Hawking, S. (1971) Gravitationally Collapsed Objects of Very Low Mass. Monthly Notices of the Royal Astronomical Society, 152, 75-78.

https://doi.org/10.1093/mnras/152.1.75

[57] Radulescu, A. and Adams, P. (2013) Hebbian Crosstalk and Input Segregation. Journal of Theoretical Biology, 337, 133-149. https://doi.org/10.1016/j.jtbi.2013.08.004

[58] ' $t$ Hooft, G.T. (2018) Time, the Arrow of Time, and Quantum Mechanics. https://arxiv.org/abs/1804.01383

[59] Donoghue, J.F., Golowich, E. and Holstein, B.R. (2014) Dynamics of the Standard Model, Vol. 35. Cambridge University Press, Cambridge. https://doi.org/10.1017/CBO9780511803512

[60] Georgi, H. (2018) Lie Algebras in Particle Physics: From Isospin to Unified Theories. CRC Press, Boca Raton.

https://www.crcpress.com/Lie-Algebras-In-Particle-Physics-from-Isospin-To-Unifi ed-Theories/Georgi/p/book/9780738202334

https://doi.org/10.1201/9780429499210 
[61] Zurek, W.H. (2003) Decoherence, Einselection, and the Quantum Origins of the Classical. Reviews of Modern Physics, 75, 715. https://doi.org/10.1103/RevModPhys.75.715

[62] Liddle, A. (2015) An Introduction to Modern Cosmology. 3rd Edition, John Wiley \& Sons, Hoboken.

https://www.wiley.com/en-gb/An+Introduction+to+Modern+Cosmology,+3rd+Edi tion-p-9781118502143

[63] Hall, G.S. (2017) General Relativity. Proceedings of the Forty Sixth Scottish Universities Summer School in Physics, Aberdeen, July 1995, 432 p. https://doi.org/10.1201/9780203753804

[64] Darwin, C. (1859) The Origin of Species. Murray, London.

[65] van Wyhe, J. (2006) The Complete Work of Charles Darwin Online. Notes and Records of the Royal Society, 60, 87-89. https://doi.org/10.1098/rsnr.2005.0128

[66] Campbell, J.O. (2016) Universal Darwinism as a Process of Bayesian Inference. Frontiers in Systems Neuroscience, 10, 49. https://doi.org/10.3389/fnsys.2016.00049

[67] Whitehead, A.N. (1929) Process and Reality. Macmillan, New York.

[68] Tammes, P.M.L. (1930) On the Origin of Number and Arrangement of the Places of Exit on the Surface of Pollen-Grains. Recueil des Travaux Botaniques Néerlandais, 27, 1-84.

[69] Tóth, L.F. (1950) Some Packing and Covering Theorems. Acta Scientiarum Mathematicarum (Szeged), 12, 62-67.

[70] Robinson, R.M. (1961) Arrangement of 24 Points on a Sphere. Mathematische Annalen, 144, 17-48. https://doi.org/10.1007/BF01396539

[71] Longuet-Higgins, M.S. (2009) Snub Polyhedra and Organic Growth. Proceedings of the Royal Society A, 465, 477-491. https://doi.org/10.1098/rspa.2008.0219

[72] Erber, T. and Hockney, G.M. (1991) Equilibrium Configurations of N Equal Charges on a Sphere. Journal of Physics A, 24, 1369-1377.

[73] Morphew, D. and Chakrabarti, D. (2018) Programming Hierarchical Self-Assembly of Colloids: Matching Stability and Accessibility. Nanoscale, 10, 13875-13882. https://doi.org/10.1039/C7NR09258J

[74] Kalinin, N., Guzmán-Sáenz, A., Prieto, Y., Shkolnikov, M., Kalinina, V. and Lupercio, E. (2018) Self-Organized Criticality and Pattern Emergence through the Lens of Tropical Geometry. Proceedings of the National Academy of Sciences of the United States of America, 115, E8135-E8142. https://doi.org/10.1073/pnas.1805847115

[75] Zurek, W.H. (2009) Quantum Darwinism. Nature Physics, 5, 181-188. https://doi.org/10.1038/nphys1202

[76] Griffiths, R.B. (2007) Types of Quantum Information. Physical Review A, 76, Article ID: 062320. https://doi.org/10.1103/PhysRevA.76.062320

[77] Pisanski, T. and Servatius, B. (2013) Graphs. In: Configurations from a Graphical Viewpoint, Birkhäuser, Boston, 15-53. https://doi.org/10.1007/978-0-8176-8364-1_2

[78] Weisstein, E.W., Snub Cubical Graph. MathWorld. A Wolfram Web Resource. http://mathworld.wolfram.com/SnubCubicalGraph.html

[79] Brinkmann, G., Coolsaet, K., Goedgebeur, J. and Mélot, H. (2013) House of Graphs: A Database of Interesting Graphs. Discrete Applied Mathematics, 161, 311-314. https://doi.org/10.1016/j.dam.2012.07.018

[80] ' $t$ Hooft, G.T. (2014) The Cellular Automaton Interpretation of Quantum Mechanics. https://arxiv.org/abs/1405.1548 
[81] nLab Authors (2019) Tetrahedral Group. http://ncatlab.org/nlab/show/tetrahedral\%20group

[82] 't Hooft, G. (1993) Dimensional Reduction in Quantum Gravity. https://arxiv.org/abs/gr-qc/9310026

[83] Thorn, C.B. (1994) Reformulating String Theory with the 1/NExpansion. https://arxiv.org/abs/hep-th/9405069

[84] Susskind, L. (1995) The World as a Hologram. Journal of Mathematical Physics, 36, 6377-6396. https://doi.org/10.1063/1.531249

[85] Maldacena, J. (1999) The Large-N Limit of Superconformal Field Theories and Supergravity. International Journal of Theoretical Physics, 38, 1113-1133. https://doi.org/10.1023/A:1026654312961

[86] Wilson, R.A. (2009) Octonions and the Leech Lattice. Journal of Algebra, 322, 2186-2190. https://doi.org/10.1016/j.jalgebra.2009.03.021

[87] Baez, J. (2002) The Octonions. Bulletin of the American Mathematical Society, 39, 145-205. https://doi.org/10.1090/S0273-0979-01-00934-X

[88] Chapline, G. (2015) Leech Lattice Extension of the Non-Linear Schrodinger Equation Theory of Einstein Spaces. https://arxiv.org/abs/1510.01350

[89] Conway, J.H. and Norton, S.P. (1979) Monstrous Moonshine. Bulletin of the London Mathematical Society, 11, 308-339. https://doi.org/10.1112/blms/11.3.308

[90] Furey, C. (2018) $S U(3)_{C} \times S U(2)_{L} \times U(1)_{Y}\left(\times U(1)_{X}\right)$ as a Symmetry of Division Algebraic Ladder Operators. The European Physical Journal C, 78, 375. https://doi.org/10.1140/epjc/s10052-018-5844-7

[91] Georgi, H. and Glashow, S.L. (1974) Unity of All Elementary-Particle Forces. Physical Review Letters, 32, 438. https://doi.org/10.1103/PhysRevLett.32.438

[92] Koca, M., Koca, N.O. and Al-Shu'eili, M. (2010) Chiral Polyhedra Derived from Coxeter Diagrams and Quaternions. https://arxiv.org/abs/1006.3149

[93] Borsten, L., Dahanayake, D., Duff, M.J., Ebrahim, H. and Rubens, W. (2009) Black Holes, Qubits and Octonions. Physics Reports, 471, 113-219. https://doi.org/10.1016/j.physrep.2008.11.002

[94] Braun, V. (2012) The 24-Cell and Calabi-Yau Threefolds with Hodge Numbers $(1,1)$. Journal of High Energy Physics, 2012, 101. https://doi.org/10.1007/JHEP05(2012)101

[95] Witten, E. (1995) String Theory Dynamics in Various Dimensions. Nuclear Physics $B, 443,85-126$. https://doi.org/10.1016/0550-3213(95)00158-O

[96] Gross, D.J., Harvey, J.A., Martinec, E. and Rohm, R. (1985) Heterotic String Theory (I). The Free Heterotic String. Nuclear Physics B, 256, 253-284. https://doi.org/10.1016/0550-3213(85)90394-3

[97] Morrison, D.R. and Vafa, C. (1996) Compactifications of F-Theory on Calabi-Yau Threefolds (I). Nuclear Physics B, 473, 74-92. https://arxiv.org/abs/hep-th/9602114 https://doi.org/10.1016/0550-3213(96)00242-8

[98] Morrison, D.R. and Vafa, C. (1996) Compactifications of F-Theory on Calabi-Yau Threefolds (II). Nuclear Physics B, 476, 437-469. https://doi.org/10.1016/0550-3213(96)00369-0

[99] Musin, O.R. (2008) The Kissing Number in Four Dimensions. Annals of Mathematics, 168, 1-32. https://doi.org/10.4007/annals.2008.168.1

[100] Weisstein, E.W., Tribonacci Constant. From MathWorld. A Wolfram Web Re- 
source. http://mathworld.wolfram.com/TribonacciConstant.html

[101] Noe, T., Piezas, T. and Weisstein, E.W., Tribonacci Number. From MathWorld. A Wolfram Web Resource. http://mathworld.wolfram.com/TribonacciNumber.html

[102] Piezas III, T. (2011) Tribonacci Constant and Pi. Article 2. A Collection of Algebraic Identities. https://sites.google.com/site/tpiezas/0012

[103] Podani, J., Kun, Á. and Szilágyi, A. (2018) How Fast Does Darwin's Elephant Population Grow? Journal of the History of Biology, 51, 259-281. https://doi.org/10.1007/s10739-017-9488-5

[104] Aghanim, N., Akrami, Y., Ashdown, M., Aumont, J., Baccigalupi, C., Ballardini, M., et al. (2018) Planck 2018 Results. VI. Cosmological Parameters. https://arxiv.org/abs/1807.06209

[105] Voorhees, P.W. (1985) The Theory of Ostwald Ripening. Journal of Statistical Physics, 38, 231-252. https://doi.org/10.1007/BF01017860

[106] Ratke, L. and Beckermann, C. (2001) Concurrent Growth and Coarsening of Spheres. Acta Materialia, 49, 4041-4054. https://doi.org/10.1016/S1359-6454(01)00286-5

[107] Garcia-Morales, V. (2015) Quantum Mechanics and the Principle of Least Radix Economy. Foundations of Physics, 45, 295-332. https://doi.org/10.1007/s10701-015-9865-x

[108] Field, R.J. and Noyes, R.M. (1974) Oscillations in Chemical Systems. IV. Limit Cycle Behavior in a Model of a Real Chemical Reaction. The Journal of Chemical Physics, 60, 1877-1884. https://doi.org/10.1063/1.1681288

[109] Fleming, P. (2017) 12.11: Oscillating Reactions. Physical Chemistry. LibreTexts. California State University, Long Beach, CA.

https://chem.libretexts.org/Bookshelves/Physical_and_Theoretical_Chemistry_Text book_Maps/Book\%3A_Physical_Chemistry_(Fleming)/12\%3A_Chemical_Kinetics _II/12.11\%3A_Oscillating_Reactions

[110] Riess, A.G., Casertano, S., Yuan, W., Macri, L., Anderson, J., MacKenty, J.W., et al. (2018) New Parallaxes of Galactic Cepheids from Spatially Scanning the Hubble Space Telescope: Implications for the Hubble Constant. The Astrophysical Journal, $855,136$.

[111] Pan, W., Baldwin, K.W., West, K.W., Pfeiffer, L.N. and Tsui, D.C. (2015) Fractional Quantum Hall Effect at Landau Level Filling $v=4 / 11$. Physical Review B, 91, Article ID: 041301. https://doi.org/10.1103/PhysRevB.91.041301

[112] Rovelli, C. (2018) Black Hole Evolution Traced Out with Loop Quantum Gravity. Physics, 11, 127. https://doi.org/10.1103/Physics.11.127

[113] Davies, P. (2019) The Demon in the Machine: How Hidden Webs of Information Are Finally Solving the Mystery of Life. Penguin, London. 